\title{
Effect of Nitrogen Fertilizer Sources and Some Biocontrol Agents on Growth, Yield and Rust Disease Incidence of Some Snap Bean Cultivars Grown in Sandy Soil
}

\author{
Manal A. Mandour and Howida A. Metwaly \\ Centre Lab. Organic Agriculture, Agricultural Research \\ Centre, Cairo, Egypt.
}

$\mathbf{T}$

HIS WORK was conducted during the two successive autumn seasons of 2012/2014 at the Experimental Farm of El-Kassasein, Hort. Res. Station, Ismaelia Governorate, Egypt. The effect of three different of nitrogen fertilizer sources, i.e., ammonium sulphate (20.5 $\% \mathrm{~N}$ ) at $390.2 \mathrm{~kg} / \mathrm{fed}$. (fed. $=0.42 \mathrm{ha}$.) botanical compost at 6.667 ton/fed. and chicken manure at 2.787 ton/fed. (each equal $80 \mathrm{~kg}$ $\mathrm{N} / \mathrm{fed}$.) and five biocontrol agents (Trichoderma harzianum, Trichoderma viride, mixture of Trichoderma harzianum + Trichoderma viride and Bacillus subtilis) were tested on growth and yield as well as rust disease incidence and severity of snap bean cultivars (Paulista and Xera) were evaluated under sandy soil conditions.

Paulista cv. gave the highest dry weight of shoots/ plant and total yield/fed. compared to Xera cultivar. Chicken manure application increased dry weight of shoots and total yield of snap bean. Foliar application with mixture of Trichoderma harzianum + Trichoderma viride increased number of both leaves and branches/ plant, dry weight of shoots/ plant, pod length and total yield/feddan. Fertilizing of Paulista cv. with chicken manure combined with foliar application with Trichoderma harzianum + Trichoderma viride increased number of both leaves and branches/ plant, plant height, dry weight of branches, leaves and shoots/ plant, pod length and total yield.

Xera cv. plants gave the lowest value of rust disease severity compared to Paulista plants. Botanical compost application recorded the minimum values of rust disease incidence and severity of snap bean plants, followed by chicken manure treatment, whereas ammonium sulphate at $390.2 \mathrm{~kg} / \mathrm{fed}$. recorded maximum values. Foliar application of snap bean plants with biocontrol agents decreased rust disease incidence and severity on leaves compared to control. Mixture of Trichoderma harzianum + Trichoderma viride decreased rust disease incidence, whereas Trichoderma harzianum decreased rust disease severity.

Keywords: Snap bean cultivars, Nitrogen fertilizer sources, Rust disease, Biocontrol agents and Yield. 
Snap bean (Phaseolus vulgaris L.) is one of the most important vegetable crops grown in Egypt not only for local consumption, but also for export purpose.

In contrast to cases where a low (N) supply predisposes crops to disease, research on snap bean crops has demonstrated that an excessive (N) supply increases disease or damage caused by some pathogens. Pathogen attack of above ground parts of the plant may be encouraged by high $(\mathrm{N})$ in the presence of low $(\mathrm{K})$ and $(\mathrm{P})$. A number of studies with cereals and other crops have shown that obligate pathogens in particular, such as Puccinia ssp. causing rust and other biotrophs, can be encouraged by a high $\mathrm{N}$ supply. Possibly excess $(\mathrm{N})$ might also favor the development of infections by obligate fungal parasites in plants although there is little evidence for this. Nitrogen supplied can be a significant factor in plant disease. A supply of ammonium- $\mathrm{N}$ may predispose plants to certain diseases, while nitrate- $\mathrm{N}$ is favorable for the development of others (Palti 1981).

In Egypt the cultivated area of green beans plants at 2012 was 58.336 fed. produced 251.279 tons with average 4.307 ton/fed (FAOSTAT, 2013) .Snap bean plants did not grow well or produce high yield under low soil $\mathrm{N}$ availability (Salinas et al., 2011).It is well known that sandy soil is low fertile, low water retention, poor soil properties, (physical, chemical and biological) and had high soil $\mathrm{pH}$. Addition of organic fertilizers can improve soil aggregation stability, soil fertility, and increase caution exchange capacity and microbe activate. Also, organic fertilizer was used to decrease soil $\mathrm{pH}$ and hence increases the availability of major and minor nutrients. It also increases the water holding capacity of soil (Tahoun et al., 2000). Convert organic nutrients forms to mineral forms, which become available to plants as slow-release fertilizers (Marschner, 1995).

Feleafel and Mirdad (2014) reported that, chicken manure (equivalent 100kg $\mathrm{N} /$ fed.) significantly increased the number of leaves and branches, pod number and pod yield of snap bean cv. Super Stryke grown in sandy soil. It also contains higher levels of relatively nutritional elements especially $\mathrm{N}$, which is essentially required for plant growth (Amanullah et al., 2007).

Rust disease caused by Uromyces appendeculatus (Pers.) is one of the important fungal diseases. Rust usually reach epidemic scale in most regions of the world, include of bean rust in Egypt (Thurston, 1998).

The use of specific biological control microorganisms that interfere with plant pathogens and pests is a nature -friendly. These approaches overcome the problems caused by chemical pesticides (Harman et al., 2004). Bacteria and fungi are involved in biocontrol activity and the fungal genus Trichoderma plays a major role in controlling the plant diseases.

Application of the fungicides is not sustainable in the long term because they pollute the environment, leave harmful residues and can lead to the development of pathogen resistant strains with frequent uses (Vinale et al., 2008).

Egypt. J. Hort. Vol. 42, No.1 (2015) 
Replacement of fungicides with biocontrol agents is an alternative manage the plant pathogens, produce safety food and reduce the environment pollution (Barakat and Al-Masri, 2005).

The use of Trichoderma spp. in agriculture can provide numerous advantages, colonization of the root and rhizosphere of plant, control several plant pathogens by different mechanisms such as parasitism, antibiosis production and induce resistance stimulation of root growth improvement plant health by promote plant growth (Harman et al., 2004).

Mixing bioagents with each others may be lead to antagonistic effect consequently decrease efficacy of treatment (Robinson et al., 2009) or lead to synergistic effect and increase the efficacy. This increase or decrease is due to harmony and compatibility between bioagents.

Bacillus subtilis Cohn. is known as effective antagonist bacteria against several plant pathogens. These antagonist acts through antibiosis, secretion of volatile toxic metabolites, destructive enzymes and competition for space and nutrition. (Intana et al., 2008) found that applications of B. subtilis are an important tool not only for organic growers, but also for conventional growers. They are using B. subtilis in integrated pest management programs allow reducing the risk of both the development of strains resistant and reduce toxic residues in the final product.

The objective of this work is to study the effect of different nitrogen fertilizer sources and some biocontrol agents on growth, yield and rust diseases of snap bean cultivars (Paulista and Xera) grown in sandy soil during autumn plantations.

\section{Material and Methods}

This work was carried out during the two successive autumn seasons of 2012/2014 in the Experimental Farm at El-Kassasein, Hort. Res. Station, Ismaelia Governorate, to study the effect of three nitrogen fertilizer sources i.e., ammonium sulphate, botanical compost and chicken manure (equivalent $80 \mathrm{~kg}$ $\mathrm{N} / \mathrm{fed}$. each) and five biocontrol agents treatments (control, Trichoderma harzianum Rifai, Trichoderma viride Harz., mixture of Trichoderma harzianum + Trichoderma viride and Bacillus subtilis Cohn. on growth, yield and leaf rust disease incidence of two snap bean cultivars (Paulista and Xera) grown under sandy soil conditions.

Seeds of snap bean cvs. Paulista and Xera were obtained from Suez Canal Company for Commercial and Agricultural Development, Giza, Egypt.

The physical and chemical properties of the soil experimental site presented in Table (a). 
TABLE (a). The physical and chemical properties of the experimental soil in 20122014.

\begin{tabular}{|l|c|c|}
\hline \multicolumn{1}{|c|}{ Soil properties } & $\mathbf{2 0 1 2}$ & $\mathbf{2 0 1 4}$ \\
\hline Physical properties & & \\
Clay \% & 1.8 & 2.8 \\
Silt \% & 1.7 & 1.6 \\
Sand \% & 95.6 & 96.5 \\
Texture & Sandy & Sandy \\
Chemical properties & & \\
E.C.(mmhos/cm)* & 2.11 & 2.16 \\
PH ** & 8.32 & 8.14 \\
Organic matter (\%) & 0.07 & 0.08 \\
Available N (ppm) & 3.53 & 3.42 \\
Available P $(\mathrm{ppm})$ & 3.52 & 3.82 \\
Available K (ppm) & 11.28 & 11.45 \\
\hline
\end{tabular}

Samples of the soil were obtained from $25 \mathrm{~cm}$ soil surface

*E.C: Electric conductivity, ** pH (1:2.5 suspension).

This experiment included 30 treatments, which were the combinations among two cultivars ( Paulista and Xera), three nitrogen source ,i.e., ammonium sulphate, $20.5 \% \mathrm{~N}(\mathrm{AS})$, botanical compost (BC) and chicken manure (ChM) and five biocontrol treatments (control, Trichoderma harzianum (T1), Trichoderma viride (T2),mixture of Trichoderma harzianum + Trichoderma viride (T1+T2) and Bacillus subtilis (BS). The amounts of added AS, BC and ChM equivalent $80 \mathrm{~kg} \mathrm{~N} /$ fed./each were about $390.2 \mathrm{~kg} / \mathrm{fed} ., 6.667 \mathrm{ton} / \mathrm{fed}$. and 2.787 ton/fed., respectively.

The chemical properties of the botanical compost and chicken manure as average two seasons are presented in Table (b).

TABLE (b). The chemical properties of the botanical compost and chicken manure as average two seasons.

\begin{tabular}{|l|c|c|}
\hline Elements & Botanical compost & Chicken manure \\
\hline Macro elements \% & 1.2 & 2.78 \\
$\mathrm{~N}$ & 0.81 & 0.96 \\
$\mathrm{P}$ & 0.24 & 1.28 \\
$\mathrm{~K}$ & & 1104 \\
Micro elements (ppm) & 250 & 210 \\
$\mathrm{Fe}$ & 146 & 209 \\
$\mathrm{Mn}$ & 105 & 413 \\
$\mathrm{Zn}$ & 50 & 35.90 \\
$\mathrm{Cu}$ & 37 & \\
Organic matter (\%) & & \\
\hline
\end{tabular}

Egypt. J. Hort. Vol. 42, No.1 (2015) 


\section{Prepration of bioagents}

Bacillus subtilis (BS), Trichoderma harzianum (T1), Trichoderma viride (T2) and mixture of both $(\mathrm{T} 1+\mathrm{T} 2)$ were obtained from Central Lab. of Organic Agriculture, ARC, Giza. Trichoderma harzianum and Trichoderma viride isolate was grown in liquid gliotixin fermentation medium (GFM) developed by (Brain and Hemming 1945) for 11 days under completer darkness conditions, at $25^{\circ} \mathrm{C}$ Bacillus subtilis isolate was grown on liquid nutrient glucose medium (NGM) developed by (Dowson, 1957) for 2 days, at $25^{\circ} \mathrm{C}$. Different bioagents were prepared as suspension at concentration of $30 \times 10^{6} / \mathrm{ml}$. Mixture of Trichoderma harzianum and Trichoderma viride was prepared by mixing the two antagonists at the rate of (1:1) to increase their activity on disease control.

The treatments were arranged in a split split plot in a complete randomized block design with three replicates. Snap bean cultivars were randomly distributed in the main plot, nitrogen fertilizers sources were randomly arranged in the sub plot and biocontrol agents treatment were randomly arranged in the sub sub plot. The experimental unit area was $12.6 \mathrm{~m}^{2}$ it contained two dripper lines $(9 \mathrm{~m}$ length and $70 \mathrm{~cm}$ wide).

Seeds of Paulista and Xera cultivars were sown in $27^{\text {th }}$ Sept. and $3^{\text {rd }}$ Oct. in both autumn experimental seasons. Seeds were sown in hills on both sides of dripper line, plants thinned after 20 days from sowing as one plant/hill. The distance between each two hills was $15 \mathrm{~cm}$.

Biocontrol agents treatment (BS, T1, T2 and T1+ T2) were sprayed two times before flowering period (at 30 and 40 days from sowing). Each plot received $2 \mathrm{~L}$ spraying suspension while, the control treatments was sprayed with water only.

\section{Data Recorded}

\section{Plant growth characters}

A sample of five plants from each experimental unit was randomly taken at 50 days after sowing to evaluate, plant height, number of leaves and number of branches/plant. Samples were oven dried at $70^{\circ} \mathrm{C}$ till constant weight to determine dry weight of plant parts, i.e., shoots (branches and leaves) and pods.

\section{Assessment of rust diseases}

A random sample of ten plants from each experimental unit were randomly taken at 50 days after sowing in both study seasons for measuring reduction of disease incidence(DI) and disease severity .

DI $\%=$ (number of leaves infected $/$ total number of leaves examined $\mathrm{x} 100)$

Disease severity was determined according to the scale reported by (Hanounik 1986), as following $1=$ No pustules or very small non sporulating flecks. $3=$ Few scattered pustules covering less than $1 \%$ of leaf area and few or no pustules on stem. $5=$ Pustules common on leaves covering $1-4 \%$ of leaf area, little defoliation and some pustules on stem. $7=$ extensive pustules on leaves 
petioles and stems covering 8-10\% of leaf area, many dead leaves and severe defoliation.

DS $\%=(\operatorname{Sum}(\mathrm{n} \mathrm{X} \mathrm{v)} / 9 \mathrm{~N}$ X 100

Where: $\mathrm{n}=$ number of plants in every grade $. \mathrm{V}=$ numerical grade.

$\mathrm{N}=$ total number of leaves examined. $9=$ maximum disease grade.

\section{Yield and its components}

Green pods of each experimental unit were harvested at proper maturity stage,(counted and weighed in all harvests till the end of experiment for calculating: yield/plot and total yield/feddan. Pod length and pod diameter were also determined in samples of pod taken from the $2^{\text {nd }}$ harvest.

\section{Statistical analysis}

Data were subjected to proper statistical analysis of variance according to Snedecor and Cochran (1980) and means separation was done according to LSD at $5 \%$ level of significance.

Plant growth

\section{Results and Discussion}

Morphological characters

The main effect

Presented data in Table 1 shows that Paulista cv. gave higher number of leaves/ plant in both seasons, whereas Xera cv. recorded the higher plants in the $1^{\text {st }}$ season only. There were no significant differences between Paulista and Xera in number of branches /plant in both seasons and plant height in the $2^{\text {nd }}$ one.

Fertilizing of snap bean plants with (ChM) at 2.787 ton/fed. (fed equal 0.42 ha) significantly increased number of leaves and branches/ plant and plant height followed by (AS) at $390.2 \mathrm{~kg} / \mathrm{fed}$. at both seasons.

The beneficial effect of organic manure on growth may be due to that organic manure improves the soil structure conditions improving its aeration which encouraged the plant roots to be well developed and increase plant growth (Cook, 1972). Chicken manure contains higher levels of nutrient elements (Amanullah et al., 2007).

These elements are essentially required for plant growth and contributed to increase the meristematic activity of the plant tissues and in building protein molecules (Marschner, 1995). There for, encourage the growth of snap bean plants through promoting the plants to generate leaves and increasing the translocation of photosynthesis.

Concerning the effect of some biocontrol agents, the obtained results in Table 1 show that foliar application of snap bean plants with mixture of $(\mathrm{T} 1+\mathrm{T} 2)$ increased number of both branches, leaves/ plant and plant height at both tested seasons, except number of leaves at the $2^{\text {nd }}$ one. These results agree with those

Egypt. J. Hort. Vol. 42, No.1 (2015) 
reported by Abd El-Hamed (2014). He found that Trichoderma harzianum increased number of branches / plant of snap bean. Isolates of several other Trichoderma spp. can reduce the severity of foliar diseases, presumably by inducing systemic resistance in plants (De Ceusterand and Hoitink 1999).

TABLE 1. Effect of cultivars, nitrogen fertilizer sources and some biocontrol agents on morphological characters of snap bean plant grown under sandy soil conditions during 2012 - 2014.

\begin{tabular}{|c|c|c|c|c|c|c|}
\hline \multirow{2}{*}{ Treatments } & \multicolumn{2}{|c|}{$\begin{array}{c}\text { Branches number / } \\
\text { plant }\end{array}$} & \multicolumn{2}{|c|}{$\begin{array}{c}\text { Leaves number / } \\
\text { plant }\end{array}$} & \multicolumn{2}{|c|}{ Plant height (cm) } \\
\hline & $\begin{array}{c}1^{\text {st }} \\
\text { season }\end{array}$ & $\begin{array}{c}2^{\text {nd }} \\
\text { season }\end{array}$ & $\begin{array}{c}1^{s t} \\
\text { season }\end{array}$ & $\begin{array}{c}2^{\text {nd }} \\
\text { season }\end{array}$ & $\begin{array}{c}1^{s t} \\
\text { season }\end{array}$ & $\begin{array}{c}2^{\text {nd }} \\
\text { season }\end{array}$ \\
\hline & \multicolumn{6}{|c|}{ Effect of cultivars } \\
\hline Paulista & 4.96 & 5.14 & 19.08 & 19.51 & 47.06 & 49.56 \\
\hline Xera & 4.98 & 4.76 & 16.56 & 16.10 & 50.86 & 50.73 \\
\hline L.S.D at $5 \%$ & N.S & N.S & 0.96 & 2.84 & 2.53 & N.S \\
\hline & \multicolumn{6}{|c|}{ Effect of nitrogen sources } \\
\hline AS & 5.08 & 4.78 & 16.01 & 16.68 & 48.91 & 49.80 \\
\hline $\mathrm{BC}$ & 4.41 & 4.58 & 14.68 & 14.70 & 46.95 & 49.38 \\
\hline ChM & 5.43 & 5.50 & 22.78 & 22.03 & 51.03 & 51.26 \\
\hline L.S.D at $5 \%$ & 0.19 & 0.16 & 0.92 & 1.10 & 1.58 & 0.59 \\
\hline & \multicolumn{6}{|c|}{ Effect of biocontrol agents } \\
\hline Control & 4.69 & 5.00 & 15.00 & 18.19 & 42.44 & 52.52 \\
\hline T1 & 5.08 & 4.83 & 17.66 & 17.41 & 49.02 & 47.58 \\
\hline $\mathrm{T} 2$ & 5.00 & 4.86 & 18.80 & 18.61 & 49.58 & 50.25 \\
\hline $\mathrm{T} 1+\mathrm{T} 2$ & 5.50 & 5.33 & 20.02 & 18.00 & 57.44 & 53.66 \\
\hline BS & 4.61 & 4.75 & 17.63 & 18.80 & 46.33 & 46.72 \\
\hline L.S.D at $5 \%$ & 0.28 & 0.37 & 1.63 & N.S & 2.22 & 2.83 \\
\hline
\end{tabular}

AS: ammonium sulphate, BC: botanical compost and ChM : chicken manure NS: not significant at $0.05 \mathrm{~T}_{1}$ : Trichoderma harzianum, $\mathrm{T}_{2}$ : Trichoderma viride and $\mathrm{BS}:$ Bacillus subtilis

\section{The dual interaction effect}

Respecting the interaction between cultivars and nitrogen fertilizer sources, the obtained results in Table 2 show that, fertilizing of Xera cv. with ChM gave the highest value of number of branches/ plant in both seasons and recorded the highest plants at the $2^{\text {nd }}$ season, whereas fertilizing of Paulista cv with ChM gave the highest number of branches /plant at the $2^{\text {nd }}$ season.

As for the interaction between cultivars and biocontrol agents, results show that, foliar application of Paulista cv. with $\mathrm{T} 1+\mathrm{T} 2$ as biocontrol agents recorded the maximum values of number of both branches and leaves/ plant at both seasons testes, whereas foliar application of Xera cv. with $\mathrm{T} 1+\mathrm{T} 2$ recorded the higher plants in the $1^{\text {st }}$ season only.

Concerning the effect of interaction between nitrogen fertilizer sources and biocontrol agents (Table 2), fertilizing of snap bean plants with ChM and foliar application with $\mathrm{T} 1+\mathrm{T} 2$ as biocontrol agent gave the highest number of branches/ plant in both seasons and number of leaves/ plant in the $1^{\text {st }}$ season and recorded the maximum values of plant height at both seasons with few exceptions. Some 
investigators explain this protection effect as antibiosis action occurred in court of infection (Matei and Matei, 2008) some other investigators reported changes in plant physiology and chemical components in plants treated with these bioagents (Hafez et al., 2012).

TABLE 2. Effect of dual interaction between cultivars and nitrogen fertilizer sources, cultivars and biocontrol agents, nitrogen fertilizers sources and biocontrol agents on morphological characters of snap bean plant grown under sandy soil conditions during 2012 - 2014.

\begin{tabular}{|c|c|c|c|c|c|c|c|}
\hline \multirow{2}{*}{\multicolumn{2}{|c|}{ Treatments }} & \multicolumn{2}{|c|}{$\begin{array}{c}\text { Branches number/ } \\
\text { plant }\end{array}$} & \multicolumn{2}{|c|}{$\begin{array}{c}\begin{array}{c}\text { Leaves number/ } \\
\text { plant }\end{array} \\
\end{array}$} & \multicolumn{2}{|c|}{ Plant height (cm) } \\
\hline & & $\begin{array}{c}1^{\text {st }} \\
\text { season }\end{array}$ & $\begin{array}{c}2^{\text {nd }} \\
\text { season }\end{array}$ & $\begin{array}{c}1^{\text {st }} \\
\text { season }\end{array}$ & $\begin{array}{c}2^{\text {nd }} \\
\text { season }\end{array}$ & $\begin{array}{c}1^{\text {st }} \\
\text { season }\end{array}$ & $\begin{array}{c}2^{\text {nd }} \\
\text { season }\end{array}$ \\
\hline & & \multicolumn{6}{|c|}{ Effect of interaction between cultivars and nitrogen sources } \\
\hline \multirow{3}{*}{ 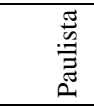 } & AS & 4.93 & 5.00 & 17.03 & 19.00 & 46.83 & 50.47 \\
\hline & $\mathrm{BC}$ & 4.60 & 5.00 & 16.10 & 16.83 & 45.90 & 49.80 \\
\hline & ChM & 5.37 & 5.43 & 24.13 & 22.70 & 48.47 & 48.43 \\
\hline \multirow{3}{*}{$\frac{\pi}{2}$} & AS & 5.23 & 4.57 & 15.00 & 14.37 & 51.00 & 49.13 \\
\hline & $\mathrm{BC}$ & 4.23 & 4.17 & 13.27 & 12.57 & 48.00 & 48.97 \\
\hline & ChM & 5.50 & 5.57 & 21.43 & 21.37 & 53.60 & 54.10 \\
\hline \multicolumn{2}{|c|}{ L.S.D at $5 \%$} & 0.02 & 0.02 & $\mathrm{NS}$ & 0.10 & NS & 0.06 \\
\hline & & \multicolumn{6}{|c|}{ Effect of interaction between cultivars and biocontrol agents } \\
\hline \multirow{5}{*}{ 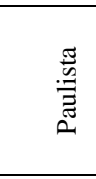 } & Control & 4.83 & 4.94 & 13.72 & 18.50 & 37.22 & 52.78 \\
\hline & $\mathrm{T} 1$ & 4.39 & 4.89 & 19.28 & 19.22 & 46.83 & 46.61 \\
\hline & $\mathrm{T} 2$ & 5.00 & 4.94 & 19.61 & 19.72 & 48.28 & 49.94 \\
\hline & $\mathrm{T} 1+\mathrm{T} 2$ & 5.06 & 5.78 & 23.06 & 20.22 & 57.00 & 51.72 \\
\hline & $\mathrm{BS}$ & 5.56 & 5.17 & 19.78 & 19.89 & 46.00 & 46.78 \\
\hline \multirow{5}{*}{$\underset{\frac{\pi}{2}}{x}$} & Control & 5.00 & 5.06 & 16.28 & 17.89 & 47.67 & 52.28 \\
\hline & $\mathrm{T} 1$ & 5.17 & 4.78 & 16.06 & 15.61 & 51.22 & 48.56 \\
\hline & $\mathrm{T} 2$ & 4.94 & 4.78 & 18.00 & 17.50 & 50.89 & 50.56 \\
\hline & $\mathrm{T} 1+\mathrm{T} 2$ & 5.44 & 4.89 & 17.00 & 15.78 & 57.89 & 55.61 \\
\hline & $\mathrm{BS}$ & 4.39 & 4.33 & 15.50 & 13.72 & 46.67 & 46.67 \\
\hline \multicolumn{2}{|c|}{ L.S.D at $5 \%$} & 0.05 & 0.06 & 0.26 & 0.27 & 0.35 & NS \\
\hline \multicolumn{8}{|c|}{ Effect of interaction between nitrogen source and biocontrol agents } \\
\hline \multirow{5}{*}{ A.S } & Control & 5.17 & 5.58 & 13.83 & 18.42 & 44.17 & 56.83 \\
\hline & T1 & 5.42 & 4.75 & 15.83 & 16.67 & 46.42 & 46.00 \\
\hline & $\mathrm{T} 2$ & 5.00 & 4.25 & 17.83 & 17.42 & 50.58 & 47.83 \\
\hline & $\mathrm{T} 1+\mathrm{T} 2$ & 5.50 & 5.20 & 18.00 & 16.17 & 56.25 & 51.50 \\
\hline & B.S & 4.33 & 4.08 & 14.58 & 14.75 & 47.17 & 46.83 \\
\hline \multirow{5}{*}{ B.C } & Control & 3.58 & 3.75 & 12.00 & 14.08 & 37.83 & 51.33 \\
\hline & $\mathrm{T} 1$ & 4.33 & 4.58 & 13.50 & 14.58 & 48.83 & 46.50 \\
\hline & $\mathrm{T} 2$ & 4.58 & 4.83 & 15.58 & 15.92 & 46.75 & 50.17 \\
\hline & $\mathrm{T} 1+\mathrm{T} 2$ & 5.25 & 5.33 & 15.00 & 14.33 & 58.17 & 53.33 \\
\hline & $\mathrm{BS}$ & 4.33 & 4.42 & 17.33 & 14.58 & 43.17 & 45.58 \\
\hline \multirow{5}{*}{$\mathrm{ChM}$} & Control & 5.33 & 5.67 & 19.17 & 22.08 & 45.33 & 49.42 \\
\hline & T1 & 5.50 & 5.17 & 23.67 & 21.00 & 51.83 & 50.25 \\
\hline & $\mathrm{T} 2$ & 5.42 & 5.50 & 23.00 & 22.50 & 51.42 & 52.75 \\
\hline & $\mathrm{T} 1+\mathrm{T} 2$ & 5.75 & 5.42 & 27.08 & 23.50 & 57.92 & 56.17 \\
\hline & $\mathrm{BS}$ & 5.17 & 5.75 & 21.00 & 21.08 & 48.67 & 47.75 \\
\hline \multicolumn{2}{|c|}{ L.S.D at $5 \%$} & 0.08 & 0.11 & 0.47 & NS & 0.64 & 0.82 \\
\hline
\end{tabular}

AS: ammonium sulphate , BC: botanical compost and ChM : chicken manure NS: not significant at $0.05 \mathrm{~T}_{1}$ : Trichoderma harzianum, $\mathrm{T}_{2}$ : Trichoderma viride, $\mathrm{BS}$ : Bacillus subtilis

Egypt. J. Hort. Vol. 42, No.1 (2015) 
The triple interaction effect

The obtained results in Table 3 indicated that, in general, the triple interaction among cvs Paulista or Xera, ChM and T1 or T2 as well as mixture of T1 + T2 gave the highest number of branches /plant, whereas the interaction among cv. Paulista, $\mathrm{ChM}$ and foliar application with $\mathrm{T} 1+\mathrm{T} 2$ as biocontrol agent recorded the maximum value of leaves's number/ plant at both seasons and plant height at the $1^{\text {st }}$ one.

TABLE 3. Effect of triple interaction among cultivars, nitrogen fertilizers sources and some biocontrol agents on morphological characters of snap bean plant grown under sandy soil conditions during 2012 - 2014.

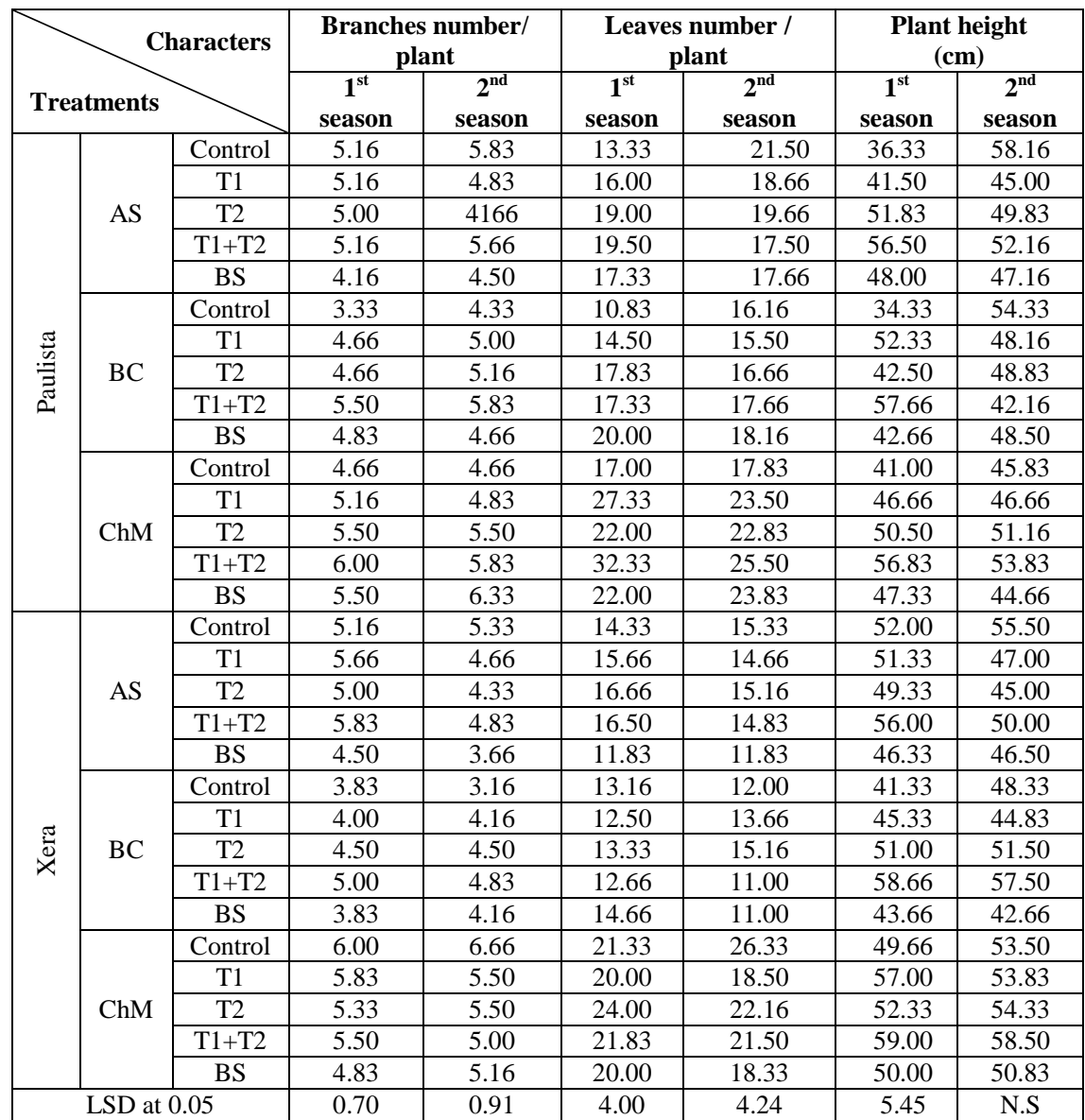

AS: ammonium sulphate, BC: botanical compost and ChM : chicken manure $\mathrm{T}_{1}$ : Trichoderma harzianum,

$\mathrm{T}_{2}$ : Trichoderma viride and $\mathrm{BS}$ : Bacillus subtilis

\section{Dry weight}

The main effect

Data presented in Table 4 showed that there were significant differences between Paulista and Xera cvs. in dry weight of branches, leaves, shoots and pods/ plant. 
Paulista cv. gave higher value of dry weight of branches and shoots, whereas Xera cv. gave higher value of dry weight of leaves and pods /plant in both seasons.

Concerning the effect of nitrogen fertilizer sources, data show that fertilization of snap bean plants with $\mathrm{ChM}$ significantly increased dry weight of branches, leaves, shoots and pods/ plant, followed by as in both seasons. Chicken manure contains several species of living organisms that release phytohormones as $\mathrm{GA}_{3}$, IAA and CYT which stimulates plant growth (Reynders and Vlassak, 1982) and this in turn increases dry matter accumulation.

Foliar application data of snap bean plants with $\mathrm{T}_{1}+\mathrm{T}_{2}$ as biocontrol agent showed an increase in dry weight of branches, leaves, shoots and pods/ plant compared to control and other biocontrol agent treatments. All biocontrol agents increased fresh and dry weight during $b$ oth seasons. This might be due to their effect on rust pathogen and have a profound effect on crop health (Kloepper et al., 2004). Abdul Wahid et al. (2007) found that application of Trichoderma as foliar application increased shoot height, number of flowers and leaf area of bbflowering and leaf expansion mechanism. There are several mechanisms to promote plant growth, the production of external growth regulators (IAA), the induction of growth regulators in treated plant and the lowering ABA content in treated plants this hormone is known to inhibit and regulate plant growth.

TABLE 4. Effect of cultivars, nitrogen fertilizer sources and some biocontrol agents on dry weight of snap bean plant grown under sandy soil conditions during 2012 - 2014.

\begin{tabular}{|c|c|c|c|c|c|c|c|c|}
\hline \multirow{2}{*}{ Treatments } & \multicolumn{2}{|c|}{$\begin{array}{c}\text { Dry weight of } \\
\text { branches/ plant (g) }\end{array}$} & \multicolumn{2}{|c|}{$\begin{array}{l}\text { Dry weight of } \\
\text { leaves/ plant (g) }\end{array}$} & \multicolumn{2}{|c|}{$\begin{array}{l}\text { Shoot dry weight } \\
\text { / plant (g) }\end{array}$} & \multicolumn{2}{|c|}{$\begin{array}{l}\text { Dry weight of } \\
\text { pods/ plant (g) }\end{array}$} \\
\hline & $\begin{array}{c}1^{\text {st }} \\
\text { Season }\end{array}$ & $\begin{array}{c}2^{\text {nd }} \\
\text { season }\end{array}$ & $\begin{array}{c}\mathbf{1}^{\text {st }} \\
\text { season }\end{array}$ & $\begin{array}{c}2^{\text {nd }} \\
\text { season }\end{array}$ & $\begin{array}{c}1^{\text {st }} \\
\text { season }\end{array}$ & $\begin{array}{c}2^{\text {nd }} \\
\text { season }\end{array}$ & $\begin{array}{c}1^{\text {st }} \\
\text { season }\end{array}$ & $\begin{array}{c}2^{n d} \\
\text { season }\end{array}$ \\
\hline & \multicolumn{8}{|c|}{ Effect of cultivars } \\
\hline Paulista & 7.39 & 7.42 & 7.91 & 7.86 & 15.30 & 15.28 & 3.96 & 3.98 \\
\hline Xera & 6.84 & 7.04 & 7.66 & 8.03 & 14.50 & 15.07 & 4.05 & 4.01 \\
\hline $\begin{array}{c}\text { L.S.D at } \\
5 \%\end{array}$ & 0.22 & & 0.06 & 0.06 & 0.04 & 0.06 & 0.64 & 0.28 \\
\hline \multicolumn{9}{|c|}{ Effect of nitrogen sources } \\
\hline AS & 7.01 & 7.02 & 7.91 & 7.72 & 14.92 & 14.74 & 4.29 & 4.22 \\
\hline $\mathrm{BC}$ & 6.03 & 5.99 & 6.36 & 6.49 & 12.39 & 12.48 & 3.33 & 3.33 \\
\hline ChM & 8.34 & 8.67 & 9.09 & 9.63 & 17.43 & 18.30 & 4.43 & 4.43 \\
\hline $\begin{array}{l}\text { L.S.D at } \\
5 \%\end{array}$ & 0.08 & 0.17 & 0.12 & 0.12 & 0.12 & 0.16 & 0.02 & 0.09 \\
\hline \multicolumn{9}{|c|}{ Effect of biocontrol agents } \\
\hline Control & 6.89 & 7.06 & 7.66 & 7.95 & 14.55 & 15.01 & 3.67 & 3.73 \\
\hline T1 & 6.84 & 7.03 & 7.61 & 7.59 & 14.45 & 14.62 & 3.82 & 3.79 \\
\hline $\mathrm{T} 2$ & 7.08 & 6.95 & 7.71 & 7.82 & 14.79 & 14.77 & 3.84 & 3.87 \\
\hline $\mathrm{T} 1+\mathrm{T} 2$ & 7.98 & 8.05 & 8.25 & 8.41 & 16.23 & 16.46 & 4.98 & 4.71 \\
\hline BS & 6.80 & 7.48 & 7.72 & 7.97 & 14.52 & 15.45 & 3.72 & 3.86 \\
\hline $\begin{array}{l}\text { L.S.D at } \\
5 \%\end{array}$ & 0.22 & 0.28 & 0.14 & 0.21 & 0.32 & 0.29 & 0.14 & 0.17 \\
\hline
\end{tabular}

AS: ammonium sulphate, BC: botanical compost and ChM : chicken manure $T_{l} \mathrm{~T}_{1}$ : Trichoderma harzianum $\mathrm{T}_{2}$ : Trichoderma viride and $\mathrm{BS}:$ Bacillus subtilis

Egypt. J. Hort. Vol. 42, No.1 (2015) 
The differences found between these cultivars might be attributed to the genetic architecture of these cultivars (Sallam, 2001).

Fertilizing snap bean plants with ChM significantly increased pod length, pod diameter, yield/ plot and total yield/fed., followed by AS treatment. The effect of compost or chicken manure fertilization might be due to the huge numbers of antagonistic microorganisms in the manure in addition to balanced nutrient substances which led to healthy plant (Hoitink, 1998).

\section{The dual interaction effect}

In general, fertilizing of Paulista cv. with ChM significantly increased dry weight of branches, leaves shoots and pods/plant, followed by fertilizing of Xera cv. with ChM Table 5.

Respecting the interaction between snap bean cultivars and foliar application with some biocontrol agents. Data revealed that no significant differences effect was found between dry weight of plant parts in both seasons, except dry weight of branches at the $1^{\text {st }}$ season. Also, the interaction between nitrogen fertilizer sources and foliar application with biocontrol agents had no significant effect on dry weight of branches, leaves shoots and pods / plant at both seasons tested, except dry weight of pods in the $1^{s t}$ seasons.

\section{The triple interaction effect}

The interaction among Paulista cv. fertilized with ChM and foliar application of $\mathrm{T} 1+\mathrm{T} 2$ as biocontrol agent gave the highest values of leaves dry weight of branches and pods/plant, followed by the interaction among Xera cv. with ChM and $\mathrm{T} 1+\mathrm{T} 2$ compared to the other triple interactions treatments (Table 6). These results may be due to that organic manures support the number of leave, fresh and dry weight $\%$ of plant through the stimulation effect on the meristematic activity of tissue where these organic manures are rich in (N.P.K) and other minerals which are compulsory for plant growth. It was also noticed that, the differences between all tested bioagents were significant in increasing the fresh and dry weight of shoots, leaves and branches when used to control rust pathogenic fungi. All biocontrol agents increased fresh and dry weight during both seasons. This might be due to their effect on rust pathogen and have a profound effect on crop health (Kloepper et al., 2004). 
TABLE 5. Effect of dual interaction between cultivars and nitrogen fertilizer sources, cultivars and biocontrol agents, nitrogen fertilizers sources and biocontrol agents on dry weight of snap bean plant grown under sandy soil conditions during 2012 - 2014.

\begin{tabular}{|c|c|c|c|c|c|c|c|c|c|}
\hline \multirow{2}{*}{\multicolumn{2}{|c|}{ Tharacters }} & \multicolumn{2}{|c|}{$\begin{array}{c}\text { Dry weight of } \\
\text { branches/ plant (g) }\end{array}$} & \multicolumn{2}{|c|}{$\begin{array}{l}\text { Dry weight of } \\
\text { leaves/ plant (g) }\end{array}$} & \multicolumn{2}{|c|}{$\begin{array}{c}\text { Shoots dry } \\
\text { weight / plant (g) }\end{array}$} & \multicolumn{2}{|c|}{\begin{tabular}{|c|} 
Dry weight of \\
pods/ plant (g)/ 30
\end{tabular}} \\
\hline & & $\begin{array}{c}1^{\text {st }} \\
\text { Season } \\
\end{array}$ & $\begin{array}{c}2^{n d} \\
\text { season }\end{array}$ & $\begin{array}{c}1^{\text {st }} \\
\text { season }\end{array}$ & $\begin{array}{c}2^{\text {nd }} \\
\text { season }\end{array}$ & $\begin{array}{c}1^{\text {st }} \\
\text { season } \\
\end{array}$ & $\begin{array}{c}2^{\text {nd }} \\
\text { season }\end{array}$ & \begin{tabular}{|c|}
$1^{s t}$ \\
season
\end{tabular} & $\begin{array}{c}2^{\text {nd }} \\
\text { season } \\
\end{array}$ \\
\hline \multicolumn{10}{|c|}{ Effect of interaction between cultivars and nitrogen sources } \\
\hline \multirow{3}{*}{ 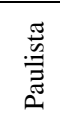 } & AS & 7.18 & 7.11 & 7.75 & 7.58 & 14.73 & 14.64 & 4.36 & 4.37 \\
\hline & $\mathrm{BC}$ & 6.05 & 6.13 & 6.74 & 6.41 & 12.79 & 12.54 & 2.89 & 2.92 \\
\hline & $\mathrm{ChM}$ & 8.94 & 9.02 & 9.25 & 9.60 & 18.19 & 18.62 & 6.45 & 4.68 \\
\hline \multirow{3}{*}{$\stackrel{\pi}{凶}$} & AS & 6.83 & 6.94 & 8.08 & 7.87 & 14.91 & 14.81 & 4.23 & 4.07 \\
\hline & $\mathrm{BC}$ & 5.95 & 5.85 & 5.97 & 6.57 & 11.92 & 12.42 & 3.71 & 3.76 \\
\hline & $\mathrm{ChM}$ & 7.75 & 8.33 & 8.92 & 9.67 & 16.67 & 18.00 & 4.22 & 4.20 \\
\hline \multicolumn{2}{|c|}{ L.S.D at $5 \%$} & 0.13 & 0.19 & 0.16 & 0.14 & 0.17 & NS & 0.07 & 0.06 \\
\hline \multicolumn{10}{|c|}{ Effect of interaction between cultivars and biocontrol agents } \\
\hline \multirow{5}{*}{ 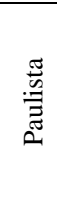 } & Control & 7.06 & 7.38 & 7.83 & 7.80 & 14.89 & 15.19 & 3.64 & 3.75 \\
\hline & $\mathrm{T} 1$ & 6.90 & 7.16 & 7.77 & 7.54 & 14.67 & 14.70 & 3.81 & 3.79 \\
\hline & $\mathrm{T} 2$ & 7.65 & 7.11 & 7.75 & 7.73 & 15.40 & 14.84 & 3.88 & 3.79 \\
\hline & $\mathrm{T} 1+\mathrm{T} 2$ & 8.38 & 8.08 & 8.41 & 8.41 & 15.73 & 15.98 & 4.85 & 4.73 \\
\hline & B.S & 6.98 & 7.36 & 7.82 & 7.83 & 14.80 & 15.19 & 3.65 & 3.85 \\
\hline \multirow{5}{*}{ 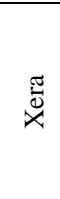 } & Control & 6.72 & 6.73 & 7.49 & 8.09 & 14.21 & 14.82 & 3.72 & 3.73 \\
\hline & $\mathrm{T} 1$ & 6.78 & 6.91 & 7.44 & 7.64 & 14.23 & 14.55 & 3.85 & 3.81 \\
\hline & $\mathrm{T} 2$ & 6.50 & 6.79 & 7.67 & 7.92 & 14.17 & 14.71 & 3.80 & 3.93 \\
\hline & $\mathrm{T} 1+\mathrm{T} 2$ & 7.58 & 8.03 & 8.09 & 8.41 & 15.67 & 15.42 & 5.12 & 4.71 \\
\hline & BS & 6.63 & 6.73 & 7.61 & 8.10 & 14.24 & 14.84 & 3.79 & 3.88 \\
\hline \multicolumn{2}{|c|}{ L.S.D at $5 \%$} & 0.39 & NS & NS & NS & NS & NS & NS & NS \\
\hline \multicolumn{10}{|c|}{ Effect of interaction between nitrogen source and biocontrol agents } \\
\hline \multirow{5}{*}{ AS } & Control & 6.73 & 6.81 & 7.74 & 7.63 & 14.47 & 14.45 & 3.98 & 3.97 \\
\hline & $\mathrm{T} 1$ & 6.86 & 6.74 & 7.74 & 7.57 & 14.60 & 14.31 & 4.09 & 3.99 \\
\hline & $\mathrm{T} 2$ & 7.02 & 6.52 & 7.88 & 7.60 & 14.90 & 14.13 & 4.07 & 4.15 \\
\hline & $\mathrm{T} 1+\mathrm{T} 2$ & 7.91 & 8.20 & 8.37 & 8.10 & 15.19 & 15.19 & 5.31 & 4.74 \\
\hline & $\mathrm{BS}$ & 6.51 & 6.85 & 7.85 & 7.71 & 14.36 & 14.56 & 4.03 & 4.18 \\
\hline \multirow{5}{*}{$\mathrm{BC}$} & Control & 5.55 & 5.69 & 6.18 & 6.58 & 11.73 & 12.27 & 2.95 & 3.13 \\
\hline & $\mathrm{T} 1$ & 5.60 & 5.90 & 6.28 & 5.97 & 11.88 & 11.87 & 3.32 & 3.21 \\
\hline & $\mathrm{T} 2$ & 6.16 & 5.82 & 6.32 & 6.27 & 12.48 & 12.09 & 3.18 & 3.24 \\
\hline & $\mathrm{T} 1+\mathrm{T} 2$ & 8.28 & 8.13 & 8.22 & 8.60 & 12.86 & 12.94 & 5.42 & 5.19 \\
\hline & BS & 5.92 & 5.79 & 6.20 & 6.58 & 12.11 & 12.37 & 2.94 & 3.24 \\
\hline \multirow{5}{*}{$\begin{array}{l}\text { Ch } \\
\mathrm{M}\end{array}$} & Control & 8.39 & 8.67 & 9.05 & 9.63 & 17.44 & 18.30 & 4.10 & 4.12 \\
\hline & $\mathrm{T} 1$ & 8.07 & 8.46 & 8.80 & 9.24 & 16.87 & 17.70 & 4.08 & 4.18 \\
\hline & $\mathrm{T} 2$ & 8.05 & 8.51 & 8.93 & 9.60 & 16.98 & 18.11 & 4.27 & 4.25 \\
\hline & $\mathrm{T} 1+\mathrm{T} 2$ & 9.24 & 9.22 & 9.55 & 10.08 & 18.13 & 18.97 & 5.52 & 5.47 \\
\hline & BS & 7.97 & 8.50 & 9.11 & 9.61 & 17.08 & 18.11 & 4.20 & 4.18 \\
\hline \multicolumn{2}{|c|}{ L.S.D at $5 \%$} & NS & NS & NS & NS & NS & NS & 0.04 & NS \\
\hline
\end{tabular}

AS: ammonium sulphate , BC: botanical compost and ChM : chicken manure NS: not significant at $0.05, \mathrm{~T}_{1}$ : Trichoderma harzianum, $\mathrm{T}_{2}$ : Trichoderma viride and $\mathrm{BS}$ : Bacillus subtilis.

Egypt. J. Hort. Vol. 42, No.1 (2015) 
EFFECT OF NITROGEN FERTILIZER SOURCES AND SOME ...

TABLE 6. Effect of triple interaction among cultivars, nitrogen fertilizers sources and some biocontrol agents on dry weight of snap bean plant grown under sandy soil conditions during 2012 - 2014.

\begin{tabular}{|c|c|c|c|c|c|c|c|c|c|c|}
\hline & \multicolumn{2}{|c|}{$\begin{array}{l}\text { Dry weight of } \\
\text { branches/ plant (g }\end{array}$} & \multicolumn{2}{|c|}{$\begin{array}{l}\text { Dry weight of } \\
\text { leaves/ plant (g) }\end{array}$} & \multicolumn{2}{|c|}{$\begin{array}{c}\text { Shoot dry weight } \\
\text { plant }(\mathrm{g})\end{array}$} & \multicolumn{2}{|c|}{$\begin{array}{l}\text { Dry weight of } \\
\text { pods/ plant (g) }\end{array}$} \\
\hline & & & \multirow{2}{*}{$\begin{array}{c}\begin{array}{c}1^{\text {st }} \\
\text { season }\end{array} \\
6.87 \\
\end{array}$} & \multirow{2}{*}{$\begin{array}{c}\begin{array}{c}\mathbf{2}^{\text {nd }} \\
\text { season }\end{array} \\
7.14\end{array}$} & \multirow{2}{*}{\begin{tabular}{c|}
$\begin{array}{c}\mathbf{1}^{\text {st }} \\
\text { season }\end{array}$ \\
7.59 \\
\end{tabular}} & \multirow{2}{*}{$\begin{array}{c}\begin{array}{c}2^{\text {nd }} \\
\text { season }\end{array} \\
7.45 \\
\end{array}$} & \multirow{2}{*}{\begin{tabular}{|c|c}
$\begin{array}{c}\mathbf{1}^{\text {st }} \\
\text { season }\end{array}$ \\
14.46 \\
\end{tabular}} & \multirow{2}{*}{$\begin{array}{c}\begin{array}{c}2^{\text {nd }} \\
\text { season }\end{array} \\
14.54 \\
\end{array}$} & \multirow{2}{*}{$\begin{array}{c}\begin{array}{c}\mathbf{1}^{\text {st }} \\
\text { season }\end{array} \\
3.95 \\
\end{array}$} & \multirow{2}{*}{$\begin{array}{c}\begin{array}{c}\mathbf{2}^{\text {nd }} \\
\text { season }\end{array} \\
4.01\end{array}$} \\
\hline \multirow{15}{*}{ 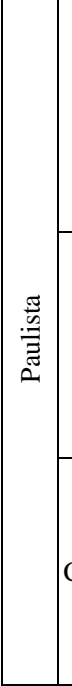 } & \multirow{5}{*}{ AS } & Control & & & & & & & & \\
\hline & & $\mathrm{T} 1$ & 6.76 & 6.74 & 7.53 & 7.47 & 14.24 & 14.21 & 4.29 & 4.08 \\
\hline & & $\mathrm{T} 2$ & 7.57 & 6.79 & 7.59 & 7.38 & 15.16 & 14.17 & 4.20 & 4.2 \\
\hline & & $\mathrm{T} 1+\mathrm{T} 2$ & 8.19 & 7.52 & 8.30 & 8.10 & 16.44 & 15.67 & 5.35 & 5.39 \\
\hline & & BS & 6.54 & 7.23 & 7.74 & 7.49 & 14.28 & 14.72 & 3.99 & 4.16 \\
\hline & \multirow{5}{*}{$\mathrm{BC}$} & Control & 5.59 & 5.92 & 6.70 & 6.36 & 12.24 & 12.28 & 2.69 & 2.84 \\
\hline & & $\mathrm{T} 1$ & 5.29 & 5.87 & 6.73 & 6.07 & 12.02 & 11.94 & 2.92 & 2.92 \\
\hline & & $\mathrm{T} 2$ & 6.37 & 5.94 & 6.65 & 6.14 & 13.02 & 12.08 & 2.91 & 2.83 \\
\hline & & $\mathrm{T} 1+\mathrm{T} 2$ & 6.99 & 6.96 & 7.06 & 6.99 & 14.05 & 13.45 & 3.31 & 3.06 \\
\hline & & $\overline{\mathrm{BS}}$ & 6.02 & 5.96 & 6.58 & 6.48 & 12.60 & 12.44 & 2.61 & 2.91 \\
\hline & \multirow{5}{*}{$\mathrm{ChM}$} & Control & 8.72 & 9.10 & 9.19 & 9.59 & 17.41 & 18.64 & 4.26 & 4.38 \\
\hline & & $\mathrm{T} 1$ & 8.65 & 8.74 & 9.06 & 9.09 & 17.71 & 17.83 & 4.20 & 4.34 \\
\hline & & $\mathrm{T} 2$ & 9.00 & 8.61 & 9.00 & 9.67 & 18.00 & 18.28 & 4.51 & 4.43 \\
\hline & & $\mathrm{T} 1+\mathrm{T} 2$ & 9.97 & 9.75 & 9.86 & 10.13 & 19.83 & 19.88 & 5.89 & 5.73 \\
\hline & & BS & 8.38 & 8.90 & 9.15 & .51 & 17.53 & 18.41 & 4.35 & 4.48 \\
\hline \multirow{15}{*}{$\stackrel{\tilde{u}}{x}$} & \multirow{5}{*}{ AS } & Control & 6.59 & 6.49 & 7.90 & 7.81 & 14.49 & 14.30 & 4.01 & 3.91 \\
\hline & & $\mathrm{T} 1$ & 6.96 & 6.61 & 7.96 & 7.66 & 14.92 & 14.27 & 3.88 & 3.90 \\
\hline & & $\mathrm{T} 2$ & 6.46 & 6.26 & 8.17 & 7.82 & 14.63 & 14.08 & 3.94 & 4.10 \\
\hline & & $\mathrm{T} 1+\mathrm{T} 2$ & 7.63 & 8.88 & 8.43 & 8.11 & 16.06 & 16.99 & 5.57 & 4.26 \\
\hline & & BS & 6.49 & 6.46 & 7.95 & 7.94 & 14.44 & 14.40 & 4.06 & 4.19 \\
\hline & \multirow{5}{*}{$\mathrm{BC}$} & Control & 5.57 & 5.46 & 5.67 & 6.80 & 11.23 & 12.26 & 3.21 & 3.41 \\
\hline & & T1 & 5.90 & 5.92 & 5.82 & 5.88 & 11.72 & 11.80 & 3.71 & 3.50 \\
\hline & & $\mathrm{T} 2$ & 5.95 & 5.71 & 5.98 & 6.24 & 11.93 & 11.95 & 3.44 & 3.64 \\
\hline & & $\mathrm{T} 1+\mathrm{T} 2$ & 6.59 & 6.51 & 6.58 & 7.09 & 13.17 & 13.60 & 4.93 & 4.65 \\
\hline & & BS & 5.83 & 5.63 & 5.81 & 6.68 & 11.64 & 12.30 & 3.26 & 3.57 \\
\hline & \multirow{5}{*}{$\mathrm{ChM}$} & Control & 8.07 & 8.24 & 8.91 & 9.67 & 16.98 & 17.91 & 3.94 & 3.84 \\
\hline & & $\mathrm{T} 1$ & 7.48 & 8.19 & 8.55 & 9.39 & 16.03 & 17.57 & 3.94 & 4.01 \\
\hline & & $\mathrm{T} 2$ & 7.10 & 8.41 & 8.86 & 9.53 & 15.96 & 17.94 & 4.02 & 4.05 \\
\hline & & $\mathrm{T} 1+\mathrm{T} 2$ & 8.52 & 8.68 & 9.24 & 10.04 & 17.76 & 18.72 & 5.15 & 5.21 \\
\hline & & BS & 7.56 & 8.11 & 9.06 & 9.70 & 16.62 & 17.81 & 4.05 & 3.87 \\
\hline \multicolumn{3}{|c|}{ LSD at 0.05} & 0.23 & 0.15 & 0.18 & 0.21 & N.S & N.S & 0.38 & 0.42 \\
\hline
\end{tabular}

AS: ammonium sulphate , BC: botanical compost and ChM : chicken manure NS: not significant at $0.05, \mathrm{~T}_{1}$ : Trichoderma harzianum, $\mathrm{T}_{2}$ : Trichoderma viride and $\mathrm{BS}:$ Bacillus subtilis. 
TABLE 7. Effect of cultivars, nitrogen fertilizer sources and some biocontrol agents on yield its components of snap been plant grown under sandy soil conditions during 2012 - 2014.

\begin{tabular}{|c|c|c|c|c|c|c|c|c|}
\hline \multirow{2}{*}{ Characters } & \multicolumn{2}{|c|}{ Pod length (cm) } & \multicolumn{2}{|c|}{ Pod diameter $(\mathrm{cm})$} & \multicolumn{2}{|c|}{ Yield /plot (kg) } & \multicolumn{2}{|c|}{ Yield /fed.(ton) } \\
\hline & $\begin{array}{c}1^{s t} \\
\text { season }\end{array}$ & $\begin{array}{c}2^{\text {nd }} \\
\text { season }\end{array}$ & $\begin{array}{c}1^{s t} \\
\text { season }\end{array}$ & $\begin{array}{c}2^{n d} \\
\text { season }\end{array}$ & $\begin{array}{c}1^{\text {st }} \\
\text { season }\end{array}$ & $\begin{array}{c}2^{\text {nd }} \\
\text { season }\end{array}$ & $\begin{array}{c}1^{\text {st }} \\
\text { season }\end{array}$ & $\begin{array}{c}2^{\text {nd }} \\
\text { season }\end{array}$ \\
\hline & \multicolumn{8}{|c|}{ Effect of cultivars } \\
\hline Paulista & 12.58 & 12.37 & 0.71 & 0.70 & 12.28 & 12.20 & 4.09 & 4.06 \\
\hline Xera & 11.68 & 11.74 & 0.70 & 0.69 & 10.87 & 10.51 & 3.62 & 3.50 \\
\hline L.S.D at $5 \%$ & 0.18 & 0.51 & N.S & N.S & 0.27 & 1.26 & 0.09 & 0.42 \\
\hline \multicolumn{9}{|c|}{ Effect of nitrogen sources } \\
\hline AS & 12.10 & 11.73 & 0.68 & 0.67 & 11.66 & 11.81 & 3.88 & 3.93 \\
\hline $\mathrm{BC}$ & 11.12 & 11.29 & 0.70 & 0.67 & 9.84 & 9.86 & 3.28 & 3.28 \\
\hline $\mathrm{ChM}$ & 13.18 & 13.15 & 0.74 & 0.76 & 13.23 & 12.40 & 4.41 & 4.13 \\
\hline L.S.D at $5 \%$ & 0.179 & 0.39 & 0.02 & 0.01 & 0.42 & 0.45 & 0.14 & 0.15 \\
\hline \multicolumn{9}{|c|}{ Effect of biocontrol agents } \\
\hline Control & 12.36 & 12.27 & 0.71 & 0.70 & 10.54 & 11.20 & 3.51 & 3.73 \\
\hline $\mathrm{T} 1$ & 11.76 & 11.62 & 0.68 & 0.67 & 10.92 & 10.55 & 3.64 & 3.51 \\
\hline $\mathrm{T} 2$ & 12.03 & 11.88 & 0.71 & 0.76 & 10.81 & 10.45 & 3.60 & 3.48 \\
\hline $\mathrm{T} 1+\mathrm{T} 2$ & 12.58 & 12.30 & 0.70 & 0.69 & 14.30 & 13.91 & 4.76 & 4.63 \\
\hline BS & 11.93 & 12.20 & 0.73 & 0.73 & 11.32 & 10.68 & 3.77 & 3.56 \\
\hline L.S.D at $5 \%$ & 0.48 & N.S & N.S & 0.03 & 0.56 & 0.80 & 0.18 & 0.26 \\
\hline
\end{tabular}

AS: ammonium sulphate, BC: botanical compost and ChM : chicken manure, $\mathrm{T}_{1}$ : Trichoderma harzianum $\mathrm{T}_{2}$ : Trichoderma viride and $\mathrm{BS}:$ Bacillus subtilis, fed $=0.42$ ha

Yield and its components

The main effect

Data in indicated that there were significant differences between Paulista and Xera cvs. in pod length, yield/ plot and total yield/fed. at both tested seasons. Paulista cv. gave higher pod length yield/ plot and total yield/fed. than Xera (Table 7).

In addition, foliar application of snap bean plants with $T_{1}+T_{2}$ as biocontrol agent increased pod length, yield/ plot and total yield/fed. These results agreed with those reported by Feleafel and Mirdad (2014) with respect to chicken manure. This improvement led to an increase in yield and dry matter. Bioagents do not only affect outside the treated plants but also affect metabolism inside plant and lead to changes in plant component (Hafez et al., 2012). In addition $B$. subtilis produce some growth regulators that increased all growth parameters compared with control treatment. (Ryder et al., 1999). On the other hand, B. subtilis grows on the treated surfaces and utilized available nutrient substances preventing pathogenic spores to establish germinate and invade healthy tissues.

\section{The dual interaction effect}

With respect to the effect of interaction between cultivars and nitrogen fertilizer sources, presented data in Table 8 show that fertilizing cv. Paulista with $\mathrm{ChM}$ recorded maximum values of pod length, yield/ plot and total yield/fed. at both seasons. Respecting the interaction between snap bean cultivars and foliar application with some biocontrol agents, the same data show that, foliar application of snap bean cv. Paulista with T1+T2 recorded maximum values of

Egypt. J. Hort. Vol. 42, No.1 (2015) 
yield/ plot and total yield/fed. in both seasons. The interaction between ChM and $\mathrm{T} 1+\mathrm{T} 2$ as biocontrol agents recorded maximum values of pod length, yield/ plot and yield/fed., whereas, the interaction between $\mathrm{ChM}$ and Bacillus subtilis (BS) as biocontrol agents recorded maximum values of pod diameter.

TABLE 8. Effect of dual interaction between cultivars and nitrogen fertilizer sources, cultivars and biocontrol agents, nitrogen fertilizers sources and biocontrol agents on yield and its components of snap bean plant grown under sandy soil conditions during 2012 - 2014.

\begin{tabular}{|c|c|c|c|c|c|c|c|c|c|}
\hline \multirow{2}{*}{\multicolumn{2}{|c|}{ Treatments }} & \multicolumn{2}{|c|}{$\begin{array}{c}\text { Pod length } \\
(\mathrm{cm})\end{array}$} & \multicolumn{2}{|c|}{$\begin{array}{c}\text { Pod diameter } \\
(\mathrm{cm})\end{array}$} & \multicolumn{2}{|c|}{$\begin{array}{c}\text { Yield /plot } \\
\text { (kg) }\end{array}$} & \multicolumn{2}{|c|}{$\begin{array}{l}\text { Yield /fed. } \\
\text { (ton) }\end{array}$} \\
\hline & & \begin{tabular}{|c|}
$1^{\text {st }}$ \\
season
\end{tabular} & $\begin{array}{c}2^{\text {nd }} \\
\text { season }\end{array}$ & $\begin{array}{c}1^{\text {st }} \\
\text { season } \\
\end{array}$ & $\begin{array}{c}2^{\text {nd }} \\
\text { season }\end{array}$ & $\begin{array}{c}1^{\text {st }} \\
\text { season }\end{array}$ & $\begin{array}{c}2^{\text {nd }} \\
\text { season } \\
\end{array}$ & $\begin{array}{c}1^{\text {st }} \\
\text { season } \\
\end{array}$ & $\begin{array}{c}2^{\text {nd }} \\
\text { season }\end{array}$ \\
\hline & & \multicolumn{8}{|c|}{ Effect of interaction between cultivars and nitrogen sources } \\
\hline \multirow{3}{*}{ 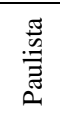 } & AS & 12.53 & 11.93 & 0.69 & 0.70 & 12.15 & 12.45 & 4.050 & 4.150 \\
\hline & $\mathrm{BC}$ & 11.80 & 11.99 & 0.71 & 0.68 & 10.90 & 10.86 & 3.633 & 3.620 \\
\hline & $\mathrm{ChM}$ & 13.43 & 13.20 & 0.74 & 0.74 & 13.82 & 13.30 & 4.607 & 4.433 \\
\hline \multirow{3}{*}{$\stackrel{\pi}{x}$} & AS & 11.67 & 11.53 & 0.68 & 0.65 & 11.19 & 11.19 & 3.730 & 3.730 \\
\hline & $\mathrm{BC}$ & 10.45 & 10.60 & 0.69 & 0.66 & 8.79 & 8.87 & 2.930 & 2.957 \\
\hline & ChM & 12.93 & 13.10 & 0.75 & 0.79 & 12.65 & 11.51 & 4.217 & 3.837 \\
\hline \multicolumn{2}{|c|}{ L.S.D at $5 \%$} & 0.02 & 0.04 & N.S & NS & 0.08 & 0.07 & 0.04 & 0.023 \\
\hline \multicolumn{10}{|c|}{ Effect of interaction between cultivars and biocontrol agents } \\
\hline \multirow{5}{*}{ 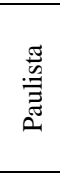 } & Control & 12.94 & 12.89 & 0.71 & 0.69 & 10.48 & 12.42 & 3.493 & 4.140 \\
\hline & T1 & 11.91 & 11.56 & 0.69 & 0.68 & 11.90 & 11.14 & 3.967 & 3.713 \\
\hline & $\mathrm{T} 2$ & 12.30 & 11.72 & 0.72 & 0.70 & 11.07 & 10.56 & 3.690 & 3.520 \\
\hline & $\mathrm{T} 1+\mathrm{T} 2$ & 13.02 & 12.51 & 0.69 & 0.67 & 15.70 & 15.13 & 5.233 & 5.043 \\
\hline & $\mathrm{BS}$ & 12.76 & 12.86 & 0.77 & 0.80 & 12.30 & 11.77 & 4.100 & 3.923 \\
\hline \multirow{5}{*}{ 苞 } & Control & \begin{tabular}{|l|}
11.78 \\
\end{tabular} & 11.67 & 0.72 & 0.71 & 10.56 & 9.98 & 3.519 & 3.327 \\
\hline & $\mathrm{T} 1$ & \begin{tabular}{|l|}
11.61 \\
\end{tabular} & 11.69 & 0.69 & 0.67 & 9.49 & 9.98 & 3.313 & 3.327 \\
\hline & $\mathrm{T} 2$ & 11.78 & 11.72 & 0.71 & 0.71 & 10.55 & 10.36 & 3.516 & 3.453 \\
\hline & $\mathrm{T} 1+\mathrm{T} 2$ & 12.14 & $\begin{array}{l}12.09 \\
\end{array}$ & 0.72 & 0.72 & 12.91 & 12.69 & 4.304 & 4.230 \\
\hline & BS & 11.11 & 11.56 & 0.70 & 0.68 & 10.34 & 9.60 & 3.446 & 3.200 \\
\hline \multicolumn{2}{|c|}{ L.S.D at $5 \%$} & N.S & N.S & N.S & N.S & 0.19 & 0.12 & 0.087 & 0.036 \\
\hline \multicolumn{10}{|c|}{ Effect of interaction between nitrogen source and biocontrol agents } \\
\hline \multirow{5}{*}{ AS } & Control & 12.67 & 12.50 & 0.73 & 0.73 & 9.68 & 11.58 & 3.227 & 3.860 \\
\hline & $\mathrm{T} 1$ & 11.92 & 11.33 & 0.63 & 0.67 & 11.09 & 10.65 & 3.697 & 3.550 \\
\hline & T2 & 12.08 & 11.67 & 0.65 & 0.62 & 11.50 & 11.57 & 3.833 & 3.857 \\
\hline & $\mathrm{T} 1+\mathrm{T} 2$ & 12.33 & 11.83 & 0.67 & 0.63 & 14.58 & 14.39 & 4.860 & 4.797 \\
\hline & BS & 11.50 & 11.33 & 0.75 & 0.72 & 11.49 & 10.90 & 3.830 & 3.633 \\
\hline \multirow{5}{*}{$\mathrm{BC}$} & Control & 11.83 & 11.50 & 0.75 & 0.67 & 8.78 & 9.98 & 2.927 & 3.327 \\
\hline & $\mathrm{T} 1$ & 10.33 & 10.70 & 0.65 & 0.62 & 8.52 & 8.57 & 2.840 & 2.857 \\
\hline & $\mathrm{T} 2$ & \begin{tabular}{|l|}
10.50 \\
\end{tabular} & 10.67 & 0.73 & 0.73 & 9.33 & 8.98 & 3.110 & 2.993 \\
\hline & $\mathrm{T} 1+\mathrm{T} 2$ & 11.38 & 11.32 & 0.72 & 0.67 & 13.54 & 13.54 & 4.513 & 4.513 \\
\hline & BS & \begin{tabular}{|l|}
11.58 \\
\end{tabular} & 12.28 & 0.67 & 0.67 & 9.94 & 9.35 & 3.164 & 3.117 \\
\hline \multirow{5}{*}{$\begin{array}{l}\text { Ch } \\
\mathrm{M}\end{array}$} & Control & 12.58 & 12.83 & 0.67 & 0.70 & 13.18 & 12.04 & 4.393 & 4.013 \\
\hline & $\mathrm{T} 1$ & 13.03 & 12.83 & 0.78 & 0.73 & 13.15 & 12.45 & 4.383 & 4.150 \\
\hline & $\mathrm{T} 2$ & 13.53 & 13.33 & 0.77 & 0.77 & 11.61 & 10.82 & 3.870 & 3.607 \\
\hline & $\mathrm{T} 1+\mathrm{T} 2$ & 14.03 & 13.75 & 0.73 & 0.78 & 15.24 & 14.91 & 5.080 & 4.970 \\
\hline & BS & 12.72 & 13.00 & 0.78 & 0.83 & 12.99 & 11.80 & 4.330 & 3.933 \\
\hline \multicolumn{2}{|c|}{ L.S.D at $5 \%$} & 0.14 & 0.16 & 0.01 & 0.01 & 0.15 & 0.22 & 0.045 & 0.066 \\
\hline
\end{tabular}

AS: ammonium sulphate , BC: botanical compost and ChM : chicken manure NS: not significant at $0.05, \mathrm{~T}_{1}$ Trichoderma harzianum, $\mathrm{T}_{2}$ : Trichoderma viride and $\mathrm{BS}:$ Bacillus subtilis, fed=.042ha. 
The triple interaction effect

The triple interaction among cultivars, nitrogen fertilizer sources and foliar application with some biocontrol agents had significant effect on pod length, pod diameter, yield/ plot and total yield/ fed. in the $2^{\text {nd }}$ season only (Table 9). The interaction among Paulista cv. $\mathrm{ChM}$ and $\mathrm{T} 1+\mathrm{T} 2$ as biocontrol agent recorded maximum values of pod length, yield/ plot and total yield/fed. without significant differences compared with the interaction.

TABLE 9. Effect of triple interaction among cultivars, nitrogen fertilizers sources and some biocontrol agents on yield and its components of snap bean plant grown under sandy soil conditions during 2012 - 2014.

\begin{tabular}{|c|c|c|c|c|c|c|c|c|c|c|}
\hline \multirow{2}{*}{\multicolumn{3}{|c|}{ Treatments }} & \multicolumn{2}{|c|}{$\begin{array}{l}\text { Pod length } \\
\text { (cm) }\end{array}$} & \multicolumn{2}{|c|}{$\begin{array}{l}\text { Pod diameter } \\
\text { (cm) }\end{array}$} & \multicolumn{2}{|c|}{$\begin{array}{c}\text { Yield /plot } \\
\text { (kg) }\end{array}$} & \multicolumn{2}{|c|}{$\begin{array}{l}\text { Yield /fed. } \\
\text { (ton) }\end{array}$} \\
\hline & & & $\begin{array}{c}1^{\text {st }} \\
\text { season }\end{array}$ & $\begin{array}{c}2^{n d} \\
\text { season }\end{array}$ & $\begin{array}{c}1^{\text {st }} \\
\text { season }\end{array}$ & $\begin{array}{c}2^{\text {nd }} \\
\text { season }\end{array}$ & $\begin{array}{c}1^{\text {st }} \\
\text { season }\end{array}$ & $\begin{array}{c}2^{\text {nd }} \\
\text { season }\end{array}$ & $\begin{array}{c}1^{s t} \\
\text { season }\end{array}$ & $\begin{array}{c}2^{\text {nd }} \\
\text { season }\end{array}$ \\
\hline \multirow{15}{*}{ 苟 } & \multirow{5}{*}{ AS } & Control & 13.33 & 13.00 & 0.70 & 0.76 & 9.36 & 13.96 & 3.12 & 4.65 \\
\hline & & T1 & 12.00 & 11.33 & 0.66 & 0.73 & 11.84 & 10.93 & 3.94 & 3.64 \\
\hline & & $\mathrm{T} 2$ & 12.66 & 12.00 & 0.66 & 0.63 & 11.93 & 11.13 & 3.97 & 3.71 \\
\hline & & $\mathrm{T} 1+\mathrm{T} 2$ & 12.33 & 11.66 & 0.66 & 0.60 & 15.14 & 14.78 & 5.04 & 4.92 \\
\hline & & BS & 12.33 & 11.66 & 0.76 & 0.76 & 12.45 & 11.43 & 4.15 & 3.81 \\
\hline & \multirow{5}{*}{$\mathrm{BC}$} & Control & 13.00 & 12.00 & 0.76 & 0.70 & 8.53 & 10.46 & 2.84 & 3.48 \\
\hline & & T1 & 10.33 & 10.66 & 0.63 & 0.63 & 9.82 & 9.44 & 3.27 & 3.14 \\
\hline & & $\mathrm{T} 2$ & 10.50 & 10.83 & 0.73 & 0.73 & 9.84 & 9.36 & 3.28 & 3.12 \\
\hline & & $\mathrm{T} 1+\mathrm{T} 2$ & 12.33 & 12.20 & 0.70 & 0.60 & 13.54 & 12.20 & 5.18 & 4.73 \\
\hline & & B.S & 12.83 & 14.23 & 0.73 & 0.73 & 10.77 & 10.83 & 3.95 & 3.61 \\
\hline & \multirow{5}{*}{$\mathrm{ChM}$} & Control & 12.50 & 13.66 & 0.66 & 0.60 & 13.53 & 12.83 & 4.51 & 4.27 \\
\hline & & T1 & 13.40 & 12.66 & 0.76 & 0.66 & 14.03 & 13.03 & 4.67 & 4.34 \\
\hline & & $\mathrm{T} 2$ & 13.73 & 13.33 & 0.76 & 0.73 & 11.43 & 11.16 & 3.81 & 3.72 \\
\hline & & $\mathrm{T} 1+\mathrm{T} 2$ & 14.40 & 13.66 & 0.70 & 0.80 & 16.41 & 15.41 & 5.47 & 5.20 \\
\hline & & BS & 13.10 & 12.66 & 0.80 & 0.90 & 13.68 & 13.03 & 4.50 & 4.34 \\
\hline \multirow{15}{*}{$\stackrel{\overrightarrow{\vec{x}}}{x}$} & \multirow{5}{*}{ AS } & Control & 12.00 & 12.00 & 0.76 & 0.70 & 10.00 & 9.2 & 3.33 & 3.06 \\
\hline & & $\mathrm{T} 1$ & 11.83 & 11.33 & 0.60 & 0.60 & 10.33 & 10.03 & 3.44 & 3.45 \\
\hline & & $\mathrm{T} 2$ & 11.50 & 11.33 & 0.63 & 0.60 & 11.06 & 12.00 & 3.68 & 4.00 \\
\hline & & $\mathrm{T} 1+\mathrm{T} 2$ & 12.33 & 12.00 & 0.66 & 0.66 & 15.00 & 14.66 & 5.00 & 4.88 \\
\hline & & BS & 10.66 & 11.00 & 0.73 & 0.66 & 10.55 & 10.26 & 3.51 & 3.45 \\
\hline & \multirow{5}{*}{$\mathrm{BC}$} & Control & 10.66 & 11.00 & 0.73 & 0.63 & 9.33 & 9.50 & 3.01 & 3.16 \\
\hline & & $\mathrm{T} 1$ & 10.33 & 10.73 & 0.66 & 0.60 & 7.22 & 7.70 & 2.40 & 2.56 \\
\hline & & $\mathrm{T} 2$ & 10.50 & 10.50 & 0.73 & 0.73 & 8.81 & 8.60 & 2.93 & 2.86 \\
\hline & & $\mathrm{T} 1+\mathrm{T} 2$ & 10.43 & 10.43 & 0.73 & 0.73 & 11.66 & 10.06 & 3.55 & 3.35 \\
\hline & & BS & 10.33 & 10.33 & 0.60 & 0.60 & 8.21 & 7.87 & 2.73 & 2.62 \\
\hline & \multirow{5}{*}{ ChM } & Control & 12.66 & 12.00 & 0.66 & 0.80 & 12.83 & 11.24 & 4.27 & 3.74 \\
\hline & & T1 & 12.66 & 13.00 & 0.80 & 0.80 & 12.26 & 11.86 & 4.08 & 3.95 \\
\hline & & $\mathrm{T} 2$ & 13.33 & 13.33 & 0.76 & 0.80 & 11.79 & 10.46 & 3.93 & 3.48 \\
\hline & & $\mathrm{T} 1+\mathrm{T} 2$ & 12.33 & 13.83 & 0.76 & 0.76 & 14.06 & 13.40 & 4.68 & 4.46 \\
\hline & & BS & 12.33 & 13.33 & 0.76 & 0.76 & 12.3 & 10.56 & 4.10 & 3.52 \\
\hline \multicolumn{3}{|c|}{ LSD at 0.05} & N.S & 1.34 & N.S & 0.08 & N.S & 2.08 & NS & 0.69 \\
\hline
\end{tabular}

AS: ammonium sulphate , BC: botanical compost and $\mathrm{ChM}$ : chicken manure NS: not significant at $0.05, \mathrm{~T}_{1}$ Trichoderma harzianum , $\mathrm{T}_{2}$ : Trichoderma viride, $\mathrm{BS}$ : Bacillus subtilis , fed=0.42ha.

Among Xera cv. ChM and T1 or T2 or T1+T2 (as biocontrol agent) with respect to pod length. On the other hand, the interaction among Paulista cv. with $\mathrm{ChM}$ and $\mathrm{BS}$ as biocontrol agent recorded maximum values of pod diameter. Increase of beans yield also due to two factors, healthy root system that can

Egypt. J. Hort. Vol. 42, No.1 (2015) 
absorb and supply adequate amount of raw nutrient substances and the syntheses of these raw nutrient material effectively in presence of high amount of chlorophyll and protein, resulted in more pod. (Sontos et al., 2001) reported that, increasing beans yield and its components as a result of chicken manure fertilization was due to the enhancing effect of chicken manure on vegetative growth.

Rust disease incidence and severity (\%)

The main effect

Presented data in Table 10 shows that, there were no significant differences between incidence (DI \%) and severity (DS \%) of rust disease, except on Paulista and Xera cultivars DS in the $2^{\text {nd }}$ season. Paulista cv. show higher rust severity $(38.79 \%)$ than Xera cv. $(30.43 \%)$ at the $2^{\text {nd }}$ season. These results agreed with those reported by Abd El-Hamed (2014) who reported that the Paulista cv. showed highest percentage of infected plants, disease severity and the lowest one in survival plants also, Xera cv. was the lowest cv. in DS and highest one in survival plants.

TABLE 10. Effect of cultivars, nitrogen fertilizer sources and some biocontrole agents on rust disease in leaves of snap been grown under sandy soil conditions during 2012 -2014.

\begin{tabular}{|c|c|c|c|c|}
\hline \multirow{3}{*}{ Treatments } & \multicolumn{4}{|c|}{ Reduction of leaf rust disease $(\%)$} \\
\hline & \multicolumn{2}{|c|}{ Incidence } & \multicolumn{2}{|c|}{ Severity } \\
\hline & $\mathbf{1}^{\text {st }}$ Season & $2^{\text {nd }}$ season & $\mathbf{1}^{\text {st }}$ season & $2^{\text {nd }}$ season \\
\hline & \multicolumn{4}{|c|}{ Effect of cultivars } \\
\hline Paulista & \multirow{2}{*}{$\begin{array}{l}21.11 \\
17.88\end{array}$} & \multirow{2}{*}{$\begin{array}{l}15.06 \\
15.66\end{array}$} & \multirow{2}{*}{$\begin{array}{l}33.97 \\
32.95\end{array}$} & \multirow{2}{*}{$\begin{array}{l}38.97 \\
30.43\end{array}$} \\
\hline Xera & & & & \\
\hline L.S.D at $5 \%$ & N.S & N.S & N.S & 1.74 \\
\hline & \multicolumn{4}{|c|}{ Effect of nitrogen sources } \\
\hline AS & 21.83 & 16.46 & 38.48 & 38.13 \\
\hline $\mathrm{BC}$ & 18.00 & 14.96 & 29.12 & 30.41 \\
\hline $\mathrm{ChM}$ & 18.66 & 14.66 & 32.79 & 35.57 \\
\hline L.S.D at $5 \%$ & 2.75 & 1.12 & 1.342 & 2.41 \\
\hline & \multicolumn{4}{|c|}{ Effect of biocontrol agents } \\
\hline Control & 28.33 & 24.72 & 48.45 & 45.11 \\
\hline $\mathrm{T} 1$ & 19.16 & 13.22 & 27.75 & 30.12 \\
\hline $\mathrm{T} 2$ & 19.44 & 14.00 & 32.98 & 35.50 \\
\hline $\mathrm{T} 1+\mathrm{T} 2$ & 15.27 & 12.33 & 30.45 & 30.74 \\
\hline BS & 15.27 & 12.55 & 27.68 & 32.05 \\
\hline L.S.D at $5 \%$ & 7.08 & 3.41 & 4.53 & 4.01 \\
\hline
\end{tabular}

AS: ammonium sulphate , BC: botanical compost and ChM : chicken manure $\mathrm{T}_{1}$ : Trichoderma harzianum, $\mathrm{T}_{2}$ : Trichoderma viride, $\mathrm{BS}$ : Bacillus subtilis. 
TABLE 11. Effect of dual interaction between cultivars and nitrogen fertilizer sources, cultivars and biocontrol agents, nitrogen fertilizers sources and biocontrol agents on rust disease in leaves of snap bean grown under sandy soil conditions during 2012-2014.

\begin{tabular}{|c|c|c|c|c|c|}
\hline \multicolumn{2}{|l|}{$\gamma^{x}$} & \multicolumn{4}{|c|}{ Reduction of leaf rust disease (\%) } \\
\hline \multirow{2}{*}{\multicolumn{2}{|c|}{ Treatments }} & \multicolumn{2}{|c|}{ Incidence } & \multicolumn{2}{|c|}{ Severity } \\
\hline & & $\begin{array}{c}1^{\text {st }} \\
\text { Season }\end{array}$ & $\begin{array}{c}2^{\text {nd }} \\
\text { season }\end{array}$ & $\begin{array}{c}1^{\text {st }} \\
\text { season }\end{array}$ & $\begin{array}{c}2^{\text {nd }} \\
\text { season }\end{array}$ \\
\hline \multicolumn{6}{|c|}{ Effect of interaction between cultivars and nitrogen sources } \\
\hline \multirow{3}{*}{ 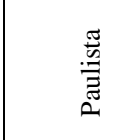 } & AS & 23.33 & 15.60 & 39.36 & 42.46 \\
\hline & $\mathrm{BC}$ & 20.00 & 14.93 & 29.12 & 34.12 \\
\hline & $\mathrm{ChM}$ & 20.00 & 14.67 & 33.45 & 40.36 \\
\hline \multirow{3}{*}{ 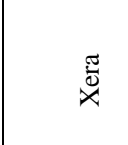 } & AS & 20.33 & 17.33 & 37.60 & 33.81 \\
\hline & $\mathrm{BC}$ & 16.00 & 15.00 & 29.14 & 26.71 \\
\hline & $\mathrm{ChM}$ & 17.33 & 14.67 & 32.13 & 30.79 \\
\hline \multicolumn{2}{|c|}{ L.S.D at $5 \%$} & N.S & N.S & N.S & N.S \\
\hline \multicolumn{6}{|c|}{ Effect of interaction between cultivars and biocontrol agents } \\
\hline \multirow{5}{*}{ 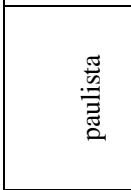 } & Control & 28.89 & 23.89 & 48.08 & 49.04 \\
\hline & $\mathrm{T} 1$ & 22.22 & 13.11 & 27.44 & 36.71 \\
\hline & $\mathrm{T} 2$ & 22.22 & 14.67 & 35.67 & 39.13 \\
\hline & $\mathrm{T} 1+\mathrm{T} 2$ & 16.67 & 11.33 & 28.06 & 34.05 \\
\hline & BS & 15.56 & 12.33 & 30.63 & 35.96 \\
\hline \multirow{5}{*}{$\stackrel{\widetilde{a}}{凶}$} & Control & 27.78 & 25.56 & 38.83 & 30.08 \\
\hline & T1 & 16.11 & 13.33 & 28.07 & 23.53 \\
\hline & $\mathrm{T} 2$ & 16.67 & 13.33 & 30.30 & 31.88 \\
\hline & $\mathrm{T} 1+\mathrm{T} 2$ & 13.89 & 13.33 & 32.85 & 27.44 \\
\hline & BS & 15.00 & 12.78 & 24.75 & 28.15 \\
\hline \multicolumn{2}{|c|}{ L.S.D at $5 \%$} & N.S & N.S & N.S & N.S \\
\hline \multicolumn{6}{|c|}{ Effect of interaction between nitrogen source and biocontrol agents } \\
\hline \multirow{5}{*}{ AS } & Control & 31.67 & 28.33 & 53.73 & 50.75 \\
\hline & T1 & 19.17 & 12.83 & 32.92 & 35.00 \\
\hline & $\mathrm{T} 2$ & 23.33 & 15.00 & 40.83 & 35.60 \\
\hline & $\mathrm{T} 1+\mathrm{T} 2$ & 18.33 & 14.17 & 32.45 & 32.07 \\
\hline & BS & 16.67 & 12.00 & 32.98 & 37.27 \\
\hline \multirow{5}{*}{$\mathrm{BC}$} & Control & 25.00 & 42.50 & 42.50 & 39.68 \\
\hline & T1 & 20.00 & 28.89 & 28.89 & 23.87 \\
\hline & $\mathrm{T} 2$ & 17.50 & 24.59 & 24.59 & 34.81 \\
\hline & $\mathrm{T} 1+\mathrm{T} 2$ & 12.50 & 25.25 & 25.20 & 28.53 \\
\hline & BS & 15.00 & 24.47 & 24.47 & 25.18 \\
\hline \multirow{5}{*}{ ChM } & Control & 28.33 & 49.14 & 49.14 & 44.93 \\
\hline & $\mathrm{T} 1$ & 18.33 & 21.97 & 21.97 & 31.50 \\
\hline & T2 & 17.50 & 33.53 & 33.53 & 36.11 \\
\hline & $\mathrm{T} 1+\mathrm{T} 2$ & 15.00 & 33.71 & 33.71 & 31.63 \\
\hline & BS & 14.17 & 25.62 & 25.62 & 33.70 \\
\hline \multicolumn{2}{|c|}{ L.S.D at 5\% } & N.S & N.S & N.S & N.S \\
\hline
\end{tabular}

Egypt. J. Hort. Vol. 42, No.1 (2015) 
Fertilizing snap bean plants with (AS) recorded maximum values of DI \% $(21.83 \%$ and $16.46 \%)$ and $(38.48 \%$ and $38.13 \%)$ for DS\% at the $1^{\text {st }}$ and $2^{\text {nd }}$ seasons, respectively. Whereas botanical compost (BC) recorded the lowest values of DI \% (18\% and $14.96 \%)$ and DS\% (29.12 and $30.41 \%)$ at the $1^{\text {st }}$ and $2^{\text {nd }}$ seasons, respectively. This might be due to that farm manure has acidic $\mathrm{pH}$. This acidic reaction makes microelements available for plant nutrition (Chaboussous, 1985).

As well as, foliar application of snap bean plants with biocontrol agents (T1 and $\mathrm{T} 2,(\mathrm{~T} 1+\mathrm{T} 2)$ and $\mathrm{BS})$ were recorded minimum values of $\mathrm{DI} \%$ and $\mathrm{DS} \%$ compared to control.

The treatment of $\mathrm{T} 1+\mathrm{T} 2$ and $\mathrm{BS}$ gave the lowest values of DI \%, while $\mathrm{T} 1$ gave the lowest values of DS\%.

These results agreed with those reported by Abd-El-Hamed (2014). He found that foliar application of snap bean with different biocontrol agents gave the lowest values of DS\% compared to control T. harzianum and Bacillus subtilis were the highest effective against the rust disease.

\section{The dual interaction effect}

The dual interactions between cultivars and nitrogen fertilizer sources, cultivars and biocontrol agents and nitrogen fertilizer source and biocontrol agents did not reflect any significant effect on leaves rust disease incidence and severity on snap bean plant at both seasons.

\section{The triple interaction effect}

The triple interaction among cultivars, nitrogen fertilizer sources and foliar application with some biocontrol agents had no significant effect on leaves rust disease incidence and severity on snap bean plant at both seasons (Table 12). The effect of mixture can be explain on the light of work carried out by (Abd El- Moity, 1985). He stated that, this synergistic effect might be due to complementary effect between different isolates included in the mixture. This means that, one isolate produced antifungal substance, whereas the second isolate has high potentialities in mycoparasitism, while the third one induce plant resistant (Bolar et al., 2000). The combination between these different effects results in high effect in controlling rust disease., whereas, the interaction between $\mathrm{ChM}$ and (BS) as biocontrol agents recorded maximum values of reduction disease incidence and disease severity (Table 12). This can be explained of the light of fact that $B$. subtilis grow very fast and occupied the court of infection and compete for spaces and nutrients preventing pathogens to invade plants (Wolk and Sorkar 1994). B. subtilis produce some growth regulators that increased all growth parameters compared with control treatment so prevent pathogens to invade plants (Ryder et al., 1999). 
TABLE 12. Effect of triple interaction among cultivars, nitrogen fertilizers sources and some biocontrol agents on rust disease in leaves of snap bean grown under sandy soil conditions during 2012-2014.

\begin{tabular}{|c|c|c|c|c|c|c|}
\hline \multicolumn{3}{|c|}{ Characters } & \multicolumn{4}{|c|}{ Reduction of leaf rust disease $(\%)$} \\
\hline \multirow{2}{*}{\multicolumn{3}{|c|}{ Treatments }} & \multicolumn{2}{|c|}{ Incidence } & \multicolumn{2}{|c|}{ Severity } \\
\hline & & & $\mathbf{1}^{s t}$ & $2^{n d}$ & $\mathbf{1}^{s t}$ & $2^{n d}$ \\
\hline \multirow{15}{*}{ 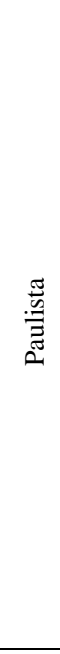 } & \multirow{5}{*}{ AS } & Control & 30.00 & 26.66 & 54.81 & 53.33 \\
\hline & & $\mathrm{T} 1$ & 23.33 & 12.33 & 31.85 & 38.88 \\
\hline & & $\mathrm{T} 2$ & 26.66 & 15.00 & 40.83 & 40.74 \\
\hline & & $\mathrm{T} 1+\mathrm{T} 2$ & 20.00 & 13.33 & 60.06 & 37.40 \\
\hline & & BS & 16.66 & 10.66 & 39.25 & 41.94 \\
\hline & \multirow{5}{*}{$\mathrm{BC}$} & Control & 26.66 & 23.33 & 40.83 & 45.18 \\
\hline & & $\mathrm{T} 1$ & 23.33 & 15.00 & 28.14 & 30.59 \\
\hline & & $\mathrm{T} 2$ & 20.00 & 14.00 & 32.85 & 37.77 \\
\hline & & $\mathrm{T} 1+\mathrm{T} 2$ & 13.33 & 11.00 & 19.29 & 30.37 \\
\hline & & $\mathrm{BS}$ & 16.66 & 12.33 & 24.47 & 26.66 \\
\hline & \multirow{5}{*}{$\begin{array}{l}\text { Ch } \\
\text { M }\end{array}$} & Control & 30.00 & 21.66 & 48.61 & 48.61 \\
\hline & & $\mathrm{T} 1$ & 20.00 & 12.00 & 22.33 & 40.66 \\
\hline & & $\mathrm{T} 2$ & 20.00 & 15.00 & 33.33 & 38.88 \\
\hline & & $\mathrm{T} 1+\mathrm{T} 2$ & 16.66 & 10.66 & 34.81 & 34.37 \\
\hline & & BS & 13.33 & 14.00 & 28.15 & 39.25 \\
\hline \multirow{15}{*}{$\stackrel{\frac{\pi}{0}}{x}$} & \multirow{5}{*}{ AS } & Control & 33.33 & 30.00 & 52.65 & 48.17 \\
\hline & & $\mathrm{T} 1$ & 15.00 & 13.33 & 32.99 & 31.11 \\
\hline & & $\mathrm{T} 2$ & 20.00 & 15.00 & 40.83 & 30.46 \\
\hline & & $\mathrm{T} 1+\mathrm{T} 2$ & 16.00 & 15.00 & 34.84 & 26.72 \\
\hline & & BS & 16.66 & 13.33 & 26.69 & 32.59 \\
\hline & \multirow{5}{*}{$\mathrm{BC}$} & Control & 23.33 & 21.66 & 44.16 & 34.16 \\
\hline & & $\mathrm{T} 1$ & 16.66 & 11.66 & 29.62 & 17.15 \\
\hline & & $\mathrm{T} 2$ & 15.00 & 13.66 & 16.33 & 31.85 \\
\hline & & $\mathrm{T} 1+\mathrm{T} 2$ & 11.66 & 15.00 & 31.11 & 26.69 \\
\hline & & BS & 13.33 & 13.33 & 24.47 & 23.00 \\
\hline & \multirow{5}{*}{$\begin{array}{l}\text { Ch } \\
\text { M }\end{array}$} & Control & 26.66 & 25.00 & 49.66 & 41.24 \\
\hline & & $\mathrm{T} 1$ & 16.66 & 15.00 & 21.59 & 22.33 \\
\hline & & $\mathrm{T} 2$ & 15.00 & 11.66 & 33.73 & 33.33 \\
\hline & & $\mathrm{T} 1+\mathrm{T} 2$ & 13.33 & 10.00 & 32.59 & 28.89 \\
\hline & & BS & 15.00 & 11.66 & 23.08 & 28.15 \\
\hline \multicolumn{3}{|c|}{ LSD at $0.05 \%$} & N.S & N.S & N.S & N.S \\
\hline
\end{tabular}

AS: ammonium sulphate , BC: botanical compost and ChM : chicken manure NS: not significant at $0.05, \mathrm{~T}_{1}$ : Trichoderma harzianum, $\mathrm{T}_{2}$ : Trichoderma viride, $\mathrm{BS}:$ Bacillus subtilis 


\section{References}

Abd-El-Hamed, W.F.M. (2014) Integrated control for white rot caused by Scelerotinia sclerotiorum (Lib) de Bary and rust caused by Uromyces appendiculatus (Pers.) Unger of bean (Phaselous vulgars L.) plant. Ph.D. Thesis, Fac. Agric., Fayoum University, Egypt.

Abd El-Moity, T.H. (1985) Effect of single and mixture of Trichoderma harzianum isolated on controlling three different soil borne pathogens. Egypt. J. Micrbiology, Special Issue., pp.111-120.

Abdul Wahid, O.A., Mustafa, A.M. and Metwally, M.R. (2007) Enchantment of plant growth through implementation of different Tricoderma species. Proceeding of the Second Enviro. Conf. Zagazig Univ., 43-59.

Amanullah, M.M., Somasundaram, E., Vaiyapuri, K. and Sathyamoorthi, K. (2007) Poultry Manure to Crops. A. Review. Agric. Rev., 28, 216-222.

Barakat, R.M. and AI- Masri, M.I. (2005) Biological control of gray mold disease (Botrytis cinerea) on tomato and bean plants by using local isolates of Trichoderma harzianum. Desert Agric. Sci., 32 (2), 145 - 156.

Brain, P.W. and Hemming, H.G. (1945) Gliotoxin a fungistatatic metabolix product of Trichoderma viride. Ann. Appl. Biol., 32, 214-220.

Bolar, J.P., Norelli, J.L. Wong, K.W., Hayes, C.K., Harman, E. and Aldwlnckle, H.S. (2000) Expression of endochitinase from Trichoderma harzianium in transgenic apple increase resistance to apple scab and duce vigour. Phytopathology, 90, 72-77.

Chaboussous, F. (1985) Sente des cultures Flammarion, Lamaison. Rustique, and Paris In: Biological control of Plant Disease Progress and Challenges for the future. Tjamos, E.C.,G.C. Papavizzas and J. Cook (Ed.) Plenum Press, New York and London published in Cooperation with NATO Scientific Affairs Division, p.4-62.

Cooke, G.W. (1972) Fertilization for Maximum Yield. Richard Clay LTD. Bungary. Suffok. Great Britian, 457p.

De Ceuster, T.J.J. and Hoitink, H.A.J. (1999) Prospects for compost sand biocontrol agents as substitutes for methyl bromide in biological control of plant diseases. Compost Sci. Util. 7, 6-15.

Dowson, W.Y. (1957) "Plant Disease Due to Bacteria", $2^{\text {nd }}$ ed., Cambridge the University Press, London, p.23.

FAOSTAT (2013) Food and Agriculture Organization of the United Nations.

Feleafel, M.N. and Mirdad, Z.M. (2014) Influence of organic nitrogen on the snap bean grown in sandy soil. International J. Agric. and Biology, 16 (1), 65-72. 
Hafez, E.E., Balbaa, M.M., Kabeil, S.S., El-Saadani, M.A. and Ahmed, S.A. (2012) Molecular studies on the biocontrol effect of Trichoderma viride and Bacillus subtilis on Fusarium oxysporum and Rhizoctonia solani infected tomato plants. World Applied Sci. J., 19 (1), 89-99.

Hanounik, S.B. (1986) Screening techniques for disease resistance in faba beans International Center for Agricultural Research in the Dry Areas (ICARDA), Alepp., Syria.

Harman, G.E., Howell, C.R., Viterbo, A., Chet, I. and Lorito, M. (2004) Trichoderma species opportunistic, avirulent plant symbionts. Nature Review, 2, $43-56$.

Hoitink, H.A.S. (1998) Opportunities for control of diseases with composts. CPP98, $7^{\text {th }}$ Internat. Cong. Pl. Pathol., 9-16 August, Edinburgh, Scotland.

Intana, W., Yenjit, P., Suwanno, T., Sattaskulchai, S., Suwanno, M. and Chamswarng, C. (2008) Efficacy of antifungal metabolites of Bacillus spp. for controlling tomato damping off caused by Pythium aphanidermatum. Walailak. J. Sci. and Tech., 5 (1), 29-38.

Kloepper, J.W., Choongmim, R.Y.U. and Zhang, S.A. (2004) Induced systemic resistance and promotion of plant growth by Bacillus spp. Phytopathology, 94(11), $1259-1266$.

Marschner, H. (1995) "Mineral Nutrition of Higher Plants", $2^{\text {nd }}$ ed., Academic Press Limeted, Text Book.

Matei, G.M. and Matei, S. (2008) Research on isolation, characterization and testing the interaction between Trichoderma harzianum and Botrytis cinerea for biological control of gray mold in strawberry. Horticultura, 51, 653-657.(CAB abstract).

Palti, J. (1981) Cultural Practices and Infectious Crop Diseases, Vol. 9. New York: Springer-Verlag.

Reynders, L. and Vlassak, K., (1982) Use of Azospirillum brasilense as biofertilizer in intensive wheat cropping. Plant and Soil, 66, 217-223.

Robinson, L., Jeger, M.J. and Xiangming, P. (2009) Management of strawberry gray mould using mixtures of biocontrol agents with different mechanisms of action. Biocontrol Sci. and Tech., 19, 1051-1065.

Ryder, M.H., Yan, Z.H., Terrace, T.E., Rovira, A.D., Tang, W., Correll, R.L., Yan, Z.H. and Tang, W. (1999) Use of strains of Bacillus isolated in China to suppress take all and Rhizabacteria root rot and to promote wheat seedling growth of glasshouse grown wheat in Australian soils. Soil Biology and Biochemistry, 31 (1), 19-29.

Salinas, R.N., Escalante, E.J.A., Rodriguez, G.M.T. and Sasa, M.E. (2011) Yield and nutritional quality of snap bean in terms of biofertilization. Trop. Subtrop. Agroecosys, 13, 347-355.

Sallam, M.E.A. (2001) Scanning micrography in evaluation of leaf rust biological control. Egypt. J. Phytopathol, 29, 11-20.

Egypt. J. Hort. Vol. 42, No.1 (2015) 
Snedecor, G.W. and Cochran, W.G. (1980) "Statistical Methods", $7^{\text {th }}$ ed., Iowa State Univ. Press, Ames, Iowa, U.S.A.

Sontos, G.M., Oliveira, A.P., Silva, J.A.L., Alves, E.U. and Costa, C.C. (2001) Characteristics and yield of snap bean pods in relation to sources and levels of organic matter. Hort. Brasileira, 19 (1), 30-35 (c.f. Hort. Abstr.71(12):10738).

Tahoun, S.A., Abdel Bary, E.A. and Atia, N.A. (2000) A greenhouse trial in view of organic forming in Egypt. J. Soil. Sci., 40 (4), $469-479$.

Thurston, D. (1998) "Tropical Plant Diseases" $2^{\text {nd }}$ ed., APS Press. The American Phytopathological Society. St. Paul, Minnesota, USA.

Vinale, F., Sivasithamparam, K., Ghisalberti, E.L., Marra, R.S.L. and Lorito, M. (2008) Trichoderma plant pathogens interactions. Soil Biology and Biochemistry, 40 - 10.

Wolk, M. and Sorkar, S. (1994) Antagonism In vivo of Bacillus spp. against Rhizocotonia solani and Pythium spp. Angeiger fur schanding skundey PF lanzenchutz, umweltschutz, 67,1-5 (c.f. Rev Pl. Path, 1994 73, 6, 4601).

(Received 14/10/2014; accepted 15/2/2015) 


\title{
تأثير بعض مصادر السماد النيتروجينى و بعض عوامل المكافحة

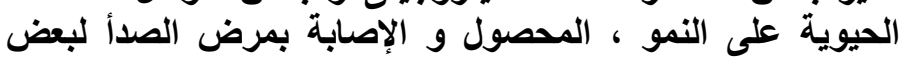 أصناف الفاصوليا المنزرعة ، الحت ظروف ول الأرض الرملية بمضية
}

\author{
منال مندور و هويدا متولى ألى ألئ

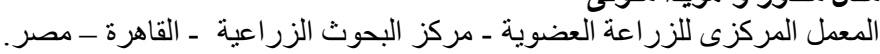

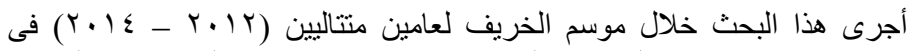

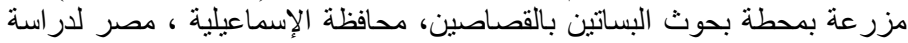

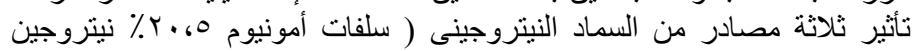

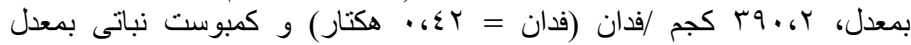
كاف

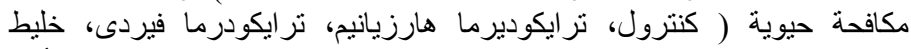

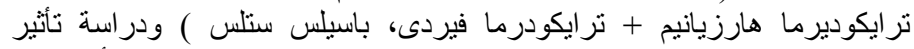

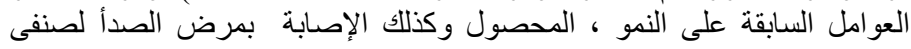

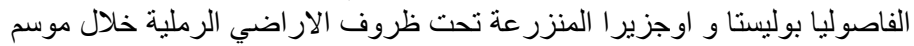
الخريف.

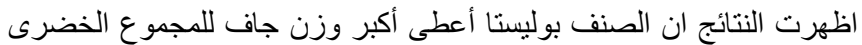

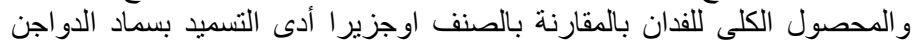

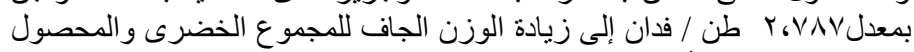

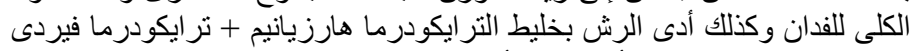

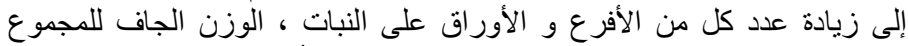

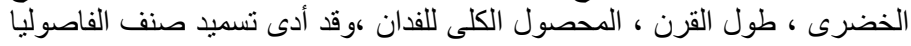

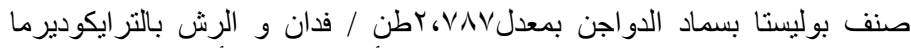

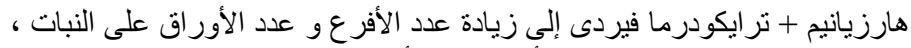

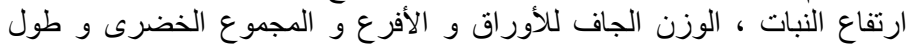

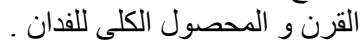

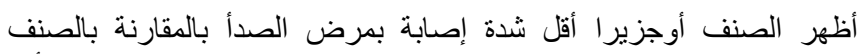

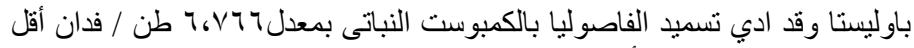

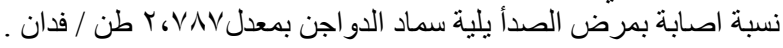

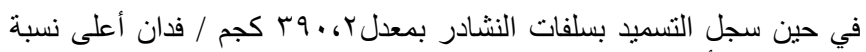

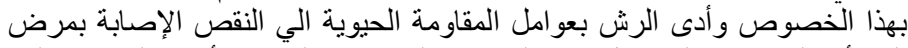

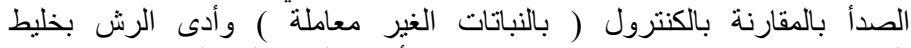

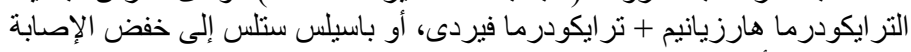
بمرض الصدأ . 